\title{
Titin truncations lead to impaired cardiomyocyte autophagy and mitochondrial function in vivo
}

Jin Zhou ${ }^{1 \#}$, Benjamin Ng ${ }^{1,2 \#}$, Nicole S J Ko ${ }^{1}$, Lorna R Fiedler ${ }^{1}$, Ester Khin ${ }^{1}$, Andrea Lim ${ }^{1}$, Norliza E $\mathrm{Sahib}^{1}$, Yajun $\mathrm{Wu}^{3}$, Sonia P Chothani ${ }^{1}$, Sebastian Schafer ${ }^{1,2}$, Boon-Huat Bay ${ }^{3}$, Rohit A. Sinha ${ }^{1,4}$, Stuart A. $\operatorname{Cook}^{1,2,5}$, Paul M. Yen ${ }^{1,6^{*}}$

${ }^{1}$ Cardiovascular \& Metabolic Disorders Program, Duke-National University of Singapore, Singapore 169857, Singapore.

${ }^{2}$ National Heart Centre Singapore, Singapore 169609, Singapore.

${ }^{3}$ Department of Anatomy, Yong Loo Lin School of Medicine, National University of Singapore, Singapore 117594, Singapore

${ }^{4}$ Department of Endocrinology, Sanjay Gandhi Postgraduate Institute of Medical Sciences, Lucknow226014, India.

${ }^{5}$ National Heart and Lung Institute, Imperial College London, London SW7 2AZ, UK.

${ }^{6}$ Duke Molecular Physiology Institute, Departments of Medicine and Pharmacology and Cancer Biology, Duke University Medical Center, Durham, NC 27710, USA.

\section{*Correspondence should be addressed to:}

Paul M. Yen (paul.yen@duke-nus.edu.sg), Cardiovascular and Metabolic Disorders Program, Duke-NUS Medical School, 8 College Road, Singapore 169857 SINGAPORE.

Tel: +65 65167332

Fax: +65 65348632

\# The authors wish it to be known that, in their opinion, the first 2 authors should be regarded as joint First Authors. 


\begin{abstract}
Titin-truncating variants (TTNtv) are the most common genetic cause of dilated cardiomyopathy (DCM). TTNtv occur in $\sim 1 \%$ of the general population and causes subclinical cardiac remodeling in asymptomatic carriers. In rat models with either proximal or distal TTNtv, we previously showed altered cardiac metabolism at baseline and impaired cardiac function in response to stress. However, the molecular mechanism(s) underlying these effects remains unknown. In the current study, we used rat models of TTNtv to investigate the effect of TTNtv on autophagy and mitochondrial function, which are essential for maintaining cellular metabolic homeostasis and cardiac function. In both the proximal and distal TTNtv rat models, we found increased levels of LC3B-II and p62 proteins, indicative of diminished autophagic degradation. The accumulation of autophagosomes and p62 protein in cardiomyocytes also was demonstrated by electron microscopy and immunochemistry, respectively. Impaired autophagy in the TTNtv heart was associated with increased phosphorylation of mTOR and decreased protein levels of the lysosomal protease, cathepsin B. In addition, TTNtv hearts showed mitochondrial dysfunction, as evidenced by decreased oxygen consumption rate (OCR) in cardiomyocytes, increased levels of reactive oxygen species (ROS), and mitochondrial protein ubiquitination. We also observed increased acetylation of mitochondrial proteins associated with decreased $\mathrm{NAD}^{+} / \mathrm{NADH}$ ratio in the TTNtv hearts. mTORC1 inhibitor, rapamycin, was able to rescue the impaired autophagy in TTNtv hearts. In summary, TTNtv leads to impaired autophagy and mitochondrial function in the heart. These changes not only provide molecular mechanisms that underlie TTNtv-associated ventricular remodeling, but also offer potential targets for its intervention.
\end{abstract}




\section{Introduction}

Dilated cardiomyopathy (DCM) is a frequent cause of heart failure that has a strong genetic basis (1). Titin-truncating variants (TTNtv) are the most common genetic variants associated with DCM. They account for approximately $25 \%$ familial DCM cases, and have high penetrance after 40 years of age $(2,3)$. Although TTNtv are seen in $\sim 1 \%$ of general population in the absence of overt DCM, sub-clinical ventricular remodeling has recently been documented showing TTNtv are penetrant but with reduced expressivity in the general population (4-7). To date, the molecular mechanism(s) underlying the pathogenesis of TTNtv-associated cardiomyopathy is not understood.

Titin is the largest human protein and spans half of the sarcomere. It contains four functionally distinct segments: (1) an N-terminus that is anchored at the Z disk; (2) a distensible I band; (3) an inextensible, thick filament-binding A band; and (4) a carboxyl M band with a kinase domain. Most DCM-associated TTNtv previously have been identified in the A band $(2,3)$, but large meta-analyses show that TTNtv encoded in constitutive exons in the Z-disc, I band, and M bands also have been associated with DCM (6).

We recently demonstrated position-independent pathogenicity of TTNtv in two distinct rat models, a distal A-band mutation model (termed TTNtvA) and a proximal Z-disc mutation model (termed TTNtvZ). Both TTNtvA and TTNtvZ rats ( $<8$ months) showed similar features of mild ventricular remodeling, and displayed slightly impaired systolic function after 1 year of age (6). Molecular profiling by genome-wide transcription and metabolomics analyses of mutant hearts revealed a shift in cardiac metabolism away from fatty acid oxidation towards glycolysis at a very early age. These functional and metabolic changes may represent maladaptive responses in TTNtv hearts (6), but their molecular basis are not known.

Although only accounts for $\sim 0.5 \%$ of body weight, the heart consumes $\sim 8 \%$ ATP produced by the body. Mitochondrial fatty acid oxidation is the predominate substrate used in the heart, being responsible for $\sim 60 \%$ of cardiac ATP production, followed by $\sim 30 \%$ contribution from glucose, $\sim 10 \%$ from lactate and ketone bodies(8). A shift of the relative contribution of these substrates has been observed under various physiological or pathological conditions(9). In heart failure, the mitochondrial oxidative capacity and fatty 
acid oxidation decline, and the failing heart switches towards glycolytic pathway $(10,11)$. This switch is most apparent in late and end-stage heart failure(12). In our animal models of TTNtv, we observed the metabolic shift towards glycolysis at a very early age, precedes an overt impairment of cardiac function (6), thus, we hypothesized that TTNtv may impair mitochondrial oxidative capacity and function which result in a switch towards glycolysis to maintain ATP production.

Autophagy is a process in which cells engulf and degrade intracellular components and cytoplasmic contents through the lysosomal pathway. This process removes macromolecules such as lipids, proteins, and glycogen to provide substrates for cellular metabolism(13), as well as damaged organelles to maintain quality control and cellular homeostasis(14). Autophagy is an essential process for normal cardiac function. Impairment of autophagy is observed in several inherited human cardiomyopathies (15-18), and pharmacological restoration of autophagy improves cardiac function $(17,18)$. Furthermore, inhibition of autophagy leads to age-related cardiomyopathy and decreased mitochondrial respiratory function in the heart (19). Currently, it is not known whether TTNtv affects cardiac autophagy or mitochondrial function. Therefore, we investigated the effect of TTNtv on cardiac autophagy and mitochondrial function to understand the mechanism(s) for pathogenicity by TTNtv.

\section{Results}

\section{Impaired autophagic degradation in TTNtv hearts}

We previously generated distal (TTNtvA, variation in the A band) and proximal (TTNtvZ, variation in Z-disc) TTNtv rat models on the same genetic background, and demonstrated the pathogenicity of TTNtv in the heart (6). To understand the molecular basis of TTNtv-triggered concentric remodeling, we examined the effect of TTNtv on autophagy. The protein levels of LC3B-II and p62, markers for autophagosome maturation and autophagic degradation, respectively, were significantly increased in hearts from both TTNtv models (Fig. 1A), whereas mRNA levels of LC3B (encoded by Maplc3b gene) and p62 (encoded by Sqstm1 gene) were unchanged (Fig. 1B and 1C). Thus, the accumulation of both LC3B-II and p62 proteins was most likely due to decreased autophagic degradation at a step downstream from LC3B-II 
incorporation into autophagosomes. We observed similar accumulation of LC3B-II and p62 in aged (48 weeks old) TTNtv rats (Fig. 1D) indicating that decreased autophagy persisted during aging. Autophagy is a homeostatic mechanism that is employed during energy stress such as starvation (13). After overnight fasting, we again observed accumulation of both p62 and LC3B-II protein (Supplementary Fig. 1), indicating there was defective autophagy during starvation. We also noted that p62 was detected as two distinct bands (Supplementary Fig. 1B). The lower band most likely represented either a splicing variant or a partially cleaved product (20). Collectively, these data showed impaired autophagic degradation in TTNtv hearts during baseline and stress conditions.

We next performed immunochemistry staining for $\mathrm{p} 62$, and found accumulation of p62 protein in cardiomyocytes of TTNtv hearts (Fig. 2A). Consistent with the increased LC3B-II protein in the TTNtv hearts, electron microscopy images showed an accumulation of autophagosomes in the cardiomyocytes (Fig. 2B-C). We also examined the expression levels of Beclin1 and ATG5, which are mainly involved in autophagosome formation, and found no significant differences between WT and TTNtv (Supplementary Fig. 2). These data further support that a downstream block in autophagy rather than decreased autophagosome formation occurred in TTNtv hearts.

We next examined the expression of lysosomal proteases in TTNtv hearts. The levels of cathepsin B was decreased in both TTNtv models, and cathepsin D was decreased in TTNtvZ hearts (Fig. 3). Decreased cathepsin B and cathepsin D protein levels in TTNtv hearts also were observed during starvation (Supplementary Figs. 3 and 4). These results indicated that impaired lysosomal degradation by proteases was associated with the decreased autophagic flux in TTNtv hearts.

To further explore whether defective lysosomal function was associated with DCM, we analyzed a published RNA-seq dataset generated from 97 end-stage DCM patients (including 7 DCM patients with TTNtv) and 105 non-diseased control samples (21). We performed differential gene expression analysis between all DCM ( $\mathrm{n}=97)$ and control, as well as DCM with TTNtv $(\mathrm{n}=7)$ and control. The expression level of TFEB, a key transcription factor for lysosome biogenesis (22), and lysosomal genes, including CTSD 
and LAMP1, were significantly down-regulated in DCM patients and DCM patients with TTNtv (Supplementary Fig. 5). These results suggested that there was lysosomal function in DCM patients.

In the heart, enhanced mTROC1 signaling may result in impaired autophagy and accumulation of p62 (17). We previously found increased mTORC1 activation in TTNtv hearts (6). To determine whether mTORC1 activation contributed to the defective autophagy in TTNtv hearts, we treated animals with mTORC1 inhibitor rapamycin. Rapamycin treatment abolished the activation of mTORC1 in TTNtv hearts (Fig. 4A and B), and increased LC3B-II and reversed p62 accumulation (Fig. 4C and D), indicating that rapamycin treatment restored autophagic flux in TTNtv hearts. These results suggested that the defective autophagy was due to mTORC1 activation.

We previously demonstrated reduced expression of full-length titin in both TTNtv models (6). To investigate whether a reduction in the amount of full-length titin could account for impaired autophagy, we used siRNA to knock down titin expression in rat cardiomyoblast H9C2 cells. Knockdown cells showed around 50\% reduction of titin mRNA expression (Fig. 5A). Autophagic flux was measured by LC3B-II accumulation with or without lysosomal inhibitor Bafilomycin A1. There was higher basal LC3B-II in knockdown cells, but lysosomal inhibition caused less accumulation of LC3B-II in titin knockdown cells than control cells treated with scrambled siRNA (Fig. 5B and C), indicating decreased autophagic flux after titin knockdown. Autophagic flux also was measured by transfecting cells with a plasmid expression a RFPGFP-LC3B fusion protein that is fluorescent for both red and green (yellow in overlaid image) in autophagosomes and only red in autolysosomes (23). As shown in Fig. 5D and E, titin knockdown cells showed significantly decreased red puncta, indicating decreased autophagic flux in titin knockdown cells. Taken together with the in vivo findings, these results supported the notion that decreased expression of titin per se was sufficient to impair autophagy.

\section{Dysfunctional/damaged mitochondria in TTNtv cardiomyocytes}

Dysfunctional mitochondria is another important contributor to the pathogenesis of heart disease (8). We previously showed that the cardiac fuel source shifted away from fatty acids towards glucose in TTNtv hearts (6), suggesting that there may be mitochondrial abnormalities in TTNtv hearts. Accordingly, we 
evaluated mitochondrial respiratory activity by measuring cellular oxygen consumption rate (OCR) in primary cardiomyocytes isolated from WT or TTNtv rats. The basal OCR, and the maximum respiratory rate were significantly lower in cardiomyocytes from TTNtv rats (Fig. 6). Knockdown of titin in H9C2 cells also showed a similar reduction of OCR (Supplementary Fig. 6), indicating that decreased expression of full-length titin led to impaired mitochondrial respiration. Since defective mitochondria can be a major source of reactive oxygen species (ROS) (8), we next examined ROS by using immunoblot-based detection of carbonyl proteins generated by excessive ROS. Increased protein carbonyls were detected in the TTNtv hearts (Supplementary Fig. 7), indicating the presence of increased ROS levels. Damaged mitochondria often exhibit increased ubiquitination of mitochondrial proteins (24). Indeed, in isolated mitochondrial fractions (Supplementary Fig. 8), we observed increased protein ubiquitination (Supplementary Fig. 9). Taken together, the co-occurrences of impaired mitochondrial respiratory activity, increased generation of ROS, increased ubiquitination of mitochondrial proteins suggested the accumulation of dysfunctional/damaged mitochondria in TTNtv hearts.

\section{Hyperacetylation of mitochondrial proteins occurs in TTNtv hearts}

Dysfunctional mitochondria and the generation of aberrant metabolites leading to hyperacetylation of cardiac mitochondrial proteins were recently described in heart failure patients (25-27). These changes likely play a causative role in the progression of the disease $(25,26)$. Thus, we examined mitochondrial protein acetylation from isolated mitochondrial fractions, and found increased acetylation of mitochondrial proteins in the TTNtv hearts (Fig. 7A-B). It previously was reported that protein hyperacetylation in the failing human heart is associated with accumulation of acetyl-CoA as well as decreased $\mathrm{NAD}^{+} / \mathrm{NADH}$ ratio $(25,27)$. The former can drive acetylation of mitochondrial proteins in the myocardium (26), whereas the latter decreases $\mathrm{NAD}^{+}$-dependent deacetylation by Sirtuin 3 (SIRT3), one of the major mitochondrial deacetylases in the heart $(25,28)$. We first examined SIRT3 protein level form isolated mitochondrial fraction, and found that mitochondrial SIRT3 level was unchanged in TTNtv hearts (Supplementary Fig. 10). To determine the levels of acetyl-CoA and $\mathrm{NAD}^{+} / \mathrm{NADH}$ ratio in the TTNtv hearts, we performed capillary electrophoresis-time of flight mass spectrometry (CE-TOFMS) analysis of these metabolites, and 
found increased acetyl-CoA level (Fig. 7C) and decreased NAD + NADH ratio in TTNtv hearts (Fig. 7D). Thus, both these changes likely contributed to the increased acetylation of mitochondrial proteins and subsequent mitochondrial dysfunction.

\section{Discussion}

In the current study, we demonstrated decreased autophagic degradation in myocardia from TTNtv rats. This was accompanied by mitochondrial abnormities, including decreased mitochondrial respiration, increased oxidative stress, and aberrant metabolites associated with increased acetylation of mitochondrial proteins. To our knowledge, this is the first demonstration of defective autophagy and dysfunctional mitochondria in TTNtv hearts. We propose that these changes may be maladaptive in TTNtv hearts.

Emerging evidence has suggested that there is an essential role for basal autophagy in maintaining normal cardiac function $(19,29)$. Here, we provided several lines of evidence that demonstrated impaired autophagic degradation in TTNtv hearts during baseline conditions. First, we found an accumulation of p62 protein in TTNtv hearts (Fig. 1, 2A, Supplementary Fig. 1). Since p62 is a scaffold protein that is subject to autophagic degradation(30), its accumulation indicated decreased autophagic flux. Of note, the accumulation of p62 protein due to impaired autophagic degradation also was reported for hereditary cardiomyopathies due to mutations in the LAMP2, MYBPC3, and LMNA genes (15-18). Second, we observed decreased levels of the lysosomal proteases, cathepsin B and D (Fig. 3, Supplementary Figs. 3 and 4), which was consistent with decreased autophagic degradation within the lysosomes of TTNtv cardiomyocytes. Third, we observed the increased accumulation of autophagosomes on electron microscopy (Fig. 2B). Next, we observed accumulation of autophagosomes without change in the protein levels of Beclin1 and ATG5 (Supplementary Fig. 2), supporting the notion that the impaired autophagy mainly was due to defective lysosomal degradation rather than decreased autophagosome formation. Taken together, these data favor a late autophagy block in TTNtv heart. Furthermore, we also observed decreased mRNA expression of key lysosomal genes in DCM patients with TTNtv, indicating that DCM might be associated with impaired lysosomal function. Of note, DCM patients without TTNtv also showed similar 
decreases in the expression of those lysosomal genes. The RNA seq analyses was performed on samples from patients with late stage DCM. Thus, it currently is unclear whether defective lysosomal function /autophagy represents a common cause for DCM or is a common late disease stage manifestation.

As the largest human protein, titin is not only responsible for the passive elasticity of the cardiac muscle, but may also function as a signaling molecule. It contains a kinase domain at Exon 358, and a few phosphorylation sites have been identified across the protein (31). We previously reported activation of mTORC1 signaling in the TTNtv heart (6), indicating a change of cellular signaling in response to TTNtv. Although the mechanistic link between TTNtv and mTOR signaling still needs further investigation, nevertheless, elevated mTORC1 signaling can negatively regulate autophagy by ULK1 phosphorylation, TFEB phosphorylation, and other unknown mechanisms (32). Indeed, activation of mTORC1 and decreased autophagy degradation has been observed in several cardiomyopathy models, and can be rescued by mTORC1 inhibition $(17,18,33)$. We also observed that administration of the mTORC1 inhibitor, rapamycin, in vivo can restore autophagy and reduce the accumulation of p62 in TTNtv hearts (Fig. 4), indicating that mTORC1 activation contributed to the impaired autophagy in TTNtv hearts.

Our previous findings showed that TTNtv hearts had reduced mitochondrial fatty acid oxidation but increased glycolysis (6), a common metabolic shift observed in cardiac hypertrophy, pressure-loaded nonfailing heart, and heart failure $(6,10,11,34,35)$. Here, in TTNtv hearts, we observed several other mitochondrial abnormalities, including decreased mitochondrial respiration, increased ROS levels and increased levels of mitochondrial protein ubiquitination (Fig. 6, Supplementary Figs. 6-9). Thus, our results support the notion that the metabolic shift in TTNtv hearts may be a maladaptive response arising from the mitochondrial dysfunction. Indeed, a recent study demonstrated altered mitochondrial energetics in TTNtv positive DCM patient (7).

In patients with heart failure and in several animal models of cardiac hypertrophy and heart failure(25, 26, 28), mitochondrial protein acetylation was found to start during cardiac hypertrophy and progressively increased with the development of heart failure (26). Significantly, we also observed increased acetylation of mitochondrial proteins in TTNtv hearts. The increased mitochondrial acetylation was likely due to a 
decrease in $\mathrm{NAD}^{+} / \mathrm{NADH}$ ratio as well as an accumulation of acetyl-CoA in TTNtv hearts (Figure 7). It is noteworthy that dysfunctional mitochondria can cause a decrease in $\mathrm{NAD}^{+} / \mathrm{NADH}$ ratio that subsequently leads to an increase in mitochondrial protein acetylation (36). Therefore, dysfunctional mitochondria in TTNtv hearts most likely led to changes in metabolite concentrations that increased mitochondrial protein acetylation. These changes could have triggered a further decline in mitochondrial function since increased lysine acetylation causes a decrease in the activity of mitochondrial enzymes $(25,26)$. Of note, nicotinamide mononucleotide $(\mathrm{NMN})$ supplementation normalizes $\mathrm{NAD}^{+} / \mathrm{NADH}$ ratio by stimulating the $\mathrm{NAD}^{+}$salvage pathway and reducing mitochondrial protein hyperacetylation, and attenuating cardiac dysfunction $(25,28)$. Therefore, our results raise the possibility that NMN supplementation may potentially decrease mitochondrial protein acetylation in TTNtv hearts and lead to beneficial outcomes in cardiomyopathy (25, 28). If so, this would represent a novel therapy to improve mitochondria quality and function in TTNtv carriers.

Although TTNtv is the most common genetic cause of DCM, most carriers are asymptomatic. However, we recently showed that eccentric cardiac remodeling occurs even in asymptomatic TTNtv carriers. Here, we showed that autophagy and mitochondrial defects are features of TTNtv in majority of rats associated with ventricular remodeling since a very early age, although these defects may not be sufficient by themselves to cause DCM in all genetic carriers. Nonetheless, it is possible that other genetic susceptibilities or an environmental, epigenetic modifier of TTN gene expression may be necessary in order to cause a silent phenotype or forme fruste to develop into the full phenotype of DCM. In summary, our results suggest that impaired autophagic degradation though activation of mTORC1, and mitochondrial dysfunction occur in TTNtv hearts. These findings provide novel insight into the molecular basis of TTNtvassociated concentric remodeling and altered metabolism as well as identify several potential novel drug targets for clinical intervention.

\section{Materials and Methods}

\section{Animal models}


Rat TTNtv models were generated as previously described (6), and maintained on a F344 background. For A-band (C-terminus) truncating variant (TTNtvA), 12bp were deleted and 2bp inserted (TA) at 228608228619 to introduce a stop codon in exon 303 (Genomic NCBI Ref Seq: NC_005102.3) corresponding to exon 327 in the human sequence. For Z-disc (N-terminus) truncating mutation (TTNtvZ) was generated by deletion of exons 2 to 6 (5286bp deletion, coordinates 2323-7608) to introduce a frame-shift.

Animal studies were conducted in accordance with the principles and procedures outlined in the National Institutes of Health Guide for the Care and Use of Laboratory Animals and were approved by the Institutional Animal Care and Use Committee (IACUC, 2013/SHS/844) at the Duke-National University of Singapore Medical School.

12-week old male rats were used for this study unless stated otherwise. For starvation studies, TTNtv and WT rats were deprived of food for 14 hours, but had free access to water. To inhibit mTOR signaling in vivo, rapamycin $(2 \mathrm{mg} / \mathrm{kg}$, dissolved in PBS containing $0.2 \%$ carboxymethyl cellulose and $0.25 \%$ tween 20) was injected intraperitoneally to TTNtv and WT rats for 7 consecutive days. To study autophagy in aged rat, 48-week old rats were used. For dissection, animal was placed in a chamber contains $70 \%$ or more $\mathrm{CO}_{2}$ (with continuous $\mathrm{CO}_{2}$ supply) till fully unconscious. Left ventricle tissue were harvested and immediately snap-frozen for subsequent western blot analysis.

\section{Western Blot Analysis}

Western blot analysis was carried out on total protein extracts from snap-frozen left ventricle tissue from 12 week old TTNtv and WT rats. Heart tissues were lysed in RIPA buffer containing protease and phosphatase inhibitors (Roche). Equal amounts of protein were separated by SDS-PAGE under reducing conditions and transferred to nitrocellulose membranes. Membranes were blocked with 5\% nonfat milk in phosphate-buffered saline with $0.1 \%$ tween 20 (PBST). The blots were incubated overnight at $4^{\circ} \mathrm{C}$ with primary antibodies. Immunoblot analysis was performed using an enhanced chemiluminescence procedure (GE Healthcare). Densitometry analysis was performed using Image J software (NIH, Bethesda, MD, USA).

\section{RNA extraction and RT-PCR}


RNA was isolated from left ventricle tissue using QIAzol (Qiagen), followed by clean-up using Invitek Mini Kit (Invitek) following the manufacturer's protocol. RNA was quantified with a Nanodrop ND-1000 spectrophotometer. $1 \mu \mathrm{g}$ of total RNA was reverse-transcribed using iSCRIPT cDNA synthesis kit (Biorad) under condtions defined by the supplier. cDNA was quantified by real-time PCR on the Rotor-Gene Q System (Qiagen). PCR was performed using QuantiFast SYBR Green PCR Kit (Qiagen) according to manufacturer's instructions.

\section{Immunohistochemistry histology}

Freshly harvested hearts from 12 weeks old TTNtv and WT rats were fixed for $24 \mathrm{~h}$ in $10 \%$ NBF, dehydrated, embedded in paraffin and $5 \mu \mathrm{m}$ sections were immunostained with anti-P62 (MBL, PM045). Primary antibody staining was visualized using ImmPRESS HRP anti-rabbit IgG polymer detection kit with ImmPACT DAB peroxidase substrate (Vector Laboratories). The sections were then counterstained with Mayer's haematoxylin (Merck).

\section{Electron Microscopy}

Fresh tissue was placed in fixative containing 2\% paraformaldehyde and 3\% gluteraldehyde in $\mathrm{pH} 7.4$ phosphate buffer overnight at $4^{\circ} \mathrm{C}$. Tissue was washed once in PBS, followed by post-fixation with $1 \%$ osmium tetroxide. Samples were dehydrated in washes with ascending concentrations of alcohol, followed by embedding in Araldite. Sections were cut and stained with uranyl acetate and lead citrate. Imaging was performed on Olympus EM208S transmission electron microscope. Autophagic vesicles were counted in 10 random fields per mouse, in 2 mice per group.

\section{Cell Culture and Transfection}

Rat cardiomyoblasts $\mathrm{H} 9 \mathrm{C} 2$ cells were purchased from ATCC and maintained at $37^{\circ} \mathrm{C}$ in DMEM containing 10\% FBS in a 5\% CO2 atmosphere. For siRNA transfection, H9C2 cells were trypsinized, mixed with opti-MEM medium (Invitrogen) containing Lipofectamine RNAimax (Invitrogen) and negative or titin siRNA according to the manufacturer's recommendations. For cDNA transfection, RFP-GFP-LC3 (tf-LC3) plasmid was transfected into H9C2 cells $48 \mathrm{~h}$ after siRNA transfection, using lipofectamine 3000 reagent (Invitrogen). 
For bafilomycin treatment, bafilomycin $(20 \mathrm{nM})$ was added to culture medium $4 \mathrm{~h}$ before protein samples were harvested.

\section{Immunofluorescence}

For autophagic flux analysis, tandem RFP-GFP-tagged LC3B plasmid was transfected into H9C2 cells with Lipofectamine 3000 Transfection Reagent (Invitrogen). $24 \mathrm{~h}$ after cDNA transfection, Cells were fixed with $4 \%$ paraformaldehyde for 30 minutes at room temperature. The nuclei were visualized by staining with $0.5 \mathrm{Ag} / \mathrm{mL}$ 4',6-diamidino-2- phenylindole (DAPI). Cells were visualized using LSM710 Carl Zeiss confocal microscope.

\section{Oxygen consumption rate (OCR) analysis in primary cardiomyocytes}

Cardiomyocytes were isolated from TTNtv rats by landendorff perfusion method as previously described(37). Briefly, rats (10-12 weeks old) were anesthetized and the heart excised and perfused with $\mathrm{Ca}^{2+}$-free Tyrodes buffer containing $0.72 \mathrm{mg} / \mathrm{ml}$ Collagenase Type I and $0.022 \mathrm{mg} / \mathrm{ml}$ Protease Type XIV (Sigma) in a retro-grade fashion via the aorta on a Langendorff apparatus for 30 minutes. After perfusion, the ventricles were meshed using a scissors and passed through a sieve to isolate cardiomyocytes. The digested materials were collected and washed in $0.2 \mathrm{mM} \mathrm{Ca}^{2+}$ Tyrodes buffer and subsequently gravitysedimented through five calcium gradients (to a final concentration of $1.25 \mathrm{mM} \mathrm{CaCl}^{2}$ ). After 30 minutes of sedimentation, the cells were pelleted, and plated at $10^{3}$ cells/well in M199 media (supplemented with $0.2 \%$ BSA, $5 \mu \mathrm{g} / \mathrm{ml}$ insulin, $5 \mathrm{mM}$ creatine, $2 \mathrm{mM}$ L-Carnitine, $5 \mathrm{mM}$ Taurine, $1 \%$ Penicillin/Streptomycin) on laminin coated seahorse XF24 tissue culture plates and allowed to attach for 2 hours. Following cell attachment, the media was changed to XF media (unbuffered DMEM supplemented with $4 \mathrm{mM}$ glutamine, $5 \mathrm{mM}$ glucose and $1 \mathrm{mM}$ pyruvate) and incubated in a non- $\mathrm{CO}_{2}$ incubator for 1 hour before the assay. Oxygen consumption rate (OCR) was measured with the Seahorse Bioscience XF24 system at 10 min intervals over a period of 2 hours. After basal respiration was measured, cardiomyocytes were subsequently treated with Oligomycin $(1 \mu \mathrm{M})$, followed by FCCP $(0.5 \mu \mathrm{M})$ and lastly Rotenone/Antimycin $\mathrm{A}(0.5 \mu \mathrm{M})$ using the XF cell mito stress kit (Seahorse Bioscience). Preliminary experiments were conducted to select optimal cell seeding density and compound concentrations according to manufacturer's instructions. 


\section{Mitochondrial fraction preparation}

Mitochondrial fraction from the left ventricle tissue was prepared using the Mitochondria/Cytosol Fractionation Kit (Abcam, ab65320) as per the manufacturer's instructions. Briefly, frozen left ventricle tissue (10 mg) was minced, homogenized in ice-cold Cytosol Extraction Buffer (containing DTT and protease inhibitors) using Dounce tissue grinder. Homogenate was transferred to a $1.5-\mathrm{ml}$ microcentrifuge tube, and centrifuge at $700 \mathrm{x}$ g for 10 minutes at $+4^{\circ} \mathrm{C}$. Supernatant was transferred to a fresh $1.5 \mathrm{ml}$ tube, and centrifuge at $10,000 \mathrm{x}$ g for 30 minutes at $+4^{\circ} \mathrm{C}$. Supernatant was collected as cytosolic fraction and pellet was collected as mitochondrial fraction.

\section{Measurement of Acetyl $\mathrm{CoA}$ and $\mathrm{NAD}^{+} / \mathrm{NADH}$ ratio}

CE-TOFMS (capillary electrophoresis-time of flight mass spectrometry) was performed to quantify $\mathrm{NAD}^{+}, \mathrm{NADH}$ and Acetyl CoA levels in heart tissue from TTNtv and WT rats as previously described(6).

\section{Statistical analysis}

Statistical analyses was performed using GraphPad Prism 6 software. ANOVA was performed for multiple experimental group comparison and P values were corrected using Dunnett's. Student t test was performed for comparison between two experimental groups. Statistical significance was determined with $\mathrm{P}<0.05$. Values of $P<0.05, P<0.01, \mathrm{P}<0.001$, and $P<0.0001$ are denoted by $*, * *, * * *$, and $* * * *$, respectively.

\section{Acknowledgements}

This work was supported by Duke-NUS Medical School Faculty Funds (to PMY), Singapore National Medical Research Council grant NMRC/CSA/0054 /2013 (to PMY), NMRC/CIRG/1340/2012 (to PMY), NMRC/STaR/011/2012 (to SAC), NMRC/STaR/0029/2017 (to SAC) and NMRC/CIRG/1450/2016 (to $\mathrm{SAC})$.

\section{Conflict of Interest Statement}

None. 


\section{Reference}

1 Hershberger, R.E., Hedges, D.J. and Morales, A. (2013) Dilated cardiomyopathy: the complexity of a diverse genetic architecture. Nat Rev Cardiol, 10, 531-547.

2 Roberts, A.M., Ware, J.S., Herman, D.S., Schafer, S., Baksi, J., Bick, A.G., Buchan, R.J., Walsh, R., John, S., Wilkinson, S. et al. (2015) Integrated allelic, transcriptional, and phenomic dissection of the cardiac effects of titin truncations in health and disease. Sci Transl Med, 7, 270 ra276.

3 Herman, D.S., Lam, L., Taylor, M.R., Wang, L., Teekakirikul, P., Christodoulou, D., Conner, L., DePalma, S.R., McDonough, B., Sparks, E. et al. (2012) Truncations of titin causing dilated cardiomyopathy. N Engl J Med, 366, 619-628.

$4 \quad$ Akinrinade, O., Koskenvuo, J.W. and Alastalo, T.P. (2015) Prevalence of Titin Truncating Variants in General Population. PLoS One, 10, e0145284.

5 Akinrinade, O., Alastalo, T.P. and Koskenvuo, J.W. (2016) Relevance of truncating titin mutations in dilated cardiomyopathy. Clin Genet, 90, 49-54.

6 Schafer, S., de Marvao, A., Adami, E., Fiedler, L.R., Ng, B., Khin, E., Rackham, O.J., van Heesch, S., Pua, C.J., Kui, M. et al. (2017) Titin-truncating variants affect heart function in disease cohorts and the general population. Nat Genet, 49, 46-53.

7 Verdonschot, J.A.J., Hazebroek, M.R., Derks, K.W.J., Barandiaran Aizpurua, A., Merken, J.J., Wang, P., Bierau, J., van den Wijngaard, A., Schalla, S.M., Abdul Hamid, M.A. et al. (2018) Titin cardiomyopathy leads to altered mitochondrial energetics, increased fibrosis and long-term lifethreatening arrhythmias. Eur Heart $J$, in press.

8 Brown, D.A., Perry, J.B., Allen, M.E., Sabbah, H.N., Stauffer, B.L., Shaikh, S.R., Cleland, J.G., Colucci, W.S., Butler, J., Voors, A.A. et al. (2017) Expert consensus document: Mitochondrial function as a therapeutic target in heart failure. Nat Rev Cardiol, 14, 238-250.

9 Fillmore, N. and Lopaschuk, G.D. (2013) Targeting mitochondrial oxidative metabolism as an approach to treat heart failure. Biochim Biophys Acta, 1833, 857-865.

10 Lai, L., Leone, T.C., Keller, M.P., Martin, O.J., Broman, A.T., Nigro, J., Kapoor, K., Koves, T.R., Stevens, R., Ilkayeva, O.R. et al. (2014) Energy metabolic reprogramming in the hypertrophied and early stage failing heart: a multisystems approach. Circ Heart Fail, 7, 1022-1031.

11 Shibayama, J., Yuzyuk, T.N., Cox, J., Makaju, A., Miller, M., Lichter, J., Li, H., Leavy, J.D., Franklin, S. and Zaitsev, A.V. (2015) Metabolic remodeling in moderate synchronous versus dyssynchronous pacing-induced heart failure: integrated metabolomics and proteomics study. PLoS One, 10, e0118974.

12 Stanley, W.C., Recchia, F.A. and Lopaschuk, G.D. (2005) Myocardial substrate metabolism in the normal and failing heart. Physiol Rev, 85, 1093-1129.

13 Rabinowitz, J.D. and White, E. (2010) Autophagy and metabolism. Science, 330, 1344-1348.

14 Sinha, R.A., Singh, B.K., Zhou, J., Wu, Y., Farah, B.L., Ohba, K., Lesmana, R., Gooding, J., Bay, B.H. and Yen, P.M. (2015) Thyroid hormone induction of mitochondrial activity is coupled to mitophagy via ROS-AMPK-ULK1 signaling. Autophagy, 11, 1341-1357. 
15 Tanaka, Y., Guhde, G., Suter, A., Eskelinen, E.L., Hartmann, D., Lullmann-Rauch, R., Janssen, P.M., Blanz, J., von Figura, K. and Saftig, P. (2000) Accumulation of autophagic vacuoles and cardiomyopathy in LAMP-2-deficient mice. Nature, 406, 902-906.

16 Nishino, I., Fu, J., Tanji, K., Yamada, T., Shimojo, S., Koori, T., Mora, M., Riggs, J.E., Oh, S.J., Koga, Y. et al. (2000) Primary LAMP-2 deficiency causes X-linked vacuolar cardiomyopathy and myopathy (Danon disease). Nature, 406, 906-910.

17 Ramos, F.J., Chen, S.C., Garelick, M.G., Dai, D.F., Liao, C.Y., Schreiber, K.H., MacKay, V.L., An, E.H., Strong, R., Ladiges, W.C. et al. (2012) Rapamycin reverses elevated mTORC1 signaling in lamin A/Cdeficient mice, rescues cardiac and skeletal muscle function, and extends survival. Sci Transl Med, 4, 144 ra103.

18 Singh, S.R., Zech, A.T.L., Geertz, B., Reischmann-Dusener, S., Osinska, H., Prondzynski, M., Kramer, E., Meng, Q., Redwood, C., van der Velden, J. et al. (2017) Activation of Autophagy Ameliorates Cardiomyopathy in Mybpc3-Targeted Knockin Mice. Circ Heart Fail, 10.

19 Taneike, M., Yamaguchi, O., Nakai, A., Hikoso, S., Takeda, T., Mizote, I., Oka, T., Tamai, T., Oyabu, J., Murakawa, T. et al. (2010) Inhibition of autophagy in the heart induces age-related cardiomyopathy. Autophagy, 6, 600-606.

20 Waguri, S. and Komatsu, M. (2009) Biochemical and morphological detection of inclusion bodies in autophagy-deficient mice. Methods Enzymol, 453, 181-196.

21 Heinig, M., Adriaens, M.E., Schafer, S., van Deutekom, H.W.M., Lodder, E.M., Ware, J.S., Schneider, V., Felkin, L.E., Creemers, E.E., Meder, B. et al. (2017) Natural genetic variation of the cardiac transcriptome in non-diseased donors and patients with dilated cardiomyopathy. Genome Biol, 18, 170.

22 Settembre, C., Di Malta, C., Polito, V.A., Garcia Arencibia, M., Vetrini, F., Erdin, S., Erdin, S.U., Huynh, T., Medina, D., Colella, P. et al. (2011) TFEB links autophagy to lysosomal biogenesis. Science, 332, 1429-1433.

23 Kimura, S., Noda, T. and Yoshimori, T. (2007) Dissection of the autophagosome maturation process by a novel reporter protein, tandem fluorescent-tagged LC3. Autophagy, 3, 452-460.

24 Escobar-Henriques, M. and Langer, T. (2014) Dynamic survey of mitochondria by ubiquitin. EMBO Rep, 15, 231-243.

25 Lee, C.F., Chavez, J.D., Garcia-Menendez, L., Choi, Y., Roe, N.D., Chiao, Y.A., Edgar, J.S., Goo, Y.A., Goodlett, D.R., Bruce, J.E. et al. (2016) Normalization of NAD+ Redox Balance as a Therapy for Heart Failure. Circulation, 134, 883-894.

26 Horton, J.L., Martin, O.J., Lai, L., Riley, N.M., Richards, A.L., Vega, R.B., Leone, T.C., Pagliarini, D.J., Muoio, D.M., Bedi, K.C., Jr. et al. (2016) Mitochondrial protein hyperacetylation in the failing heart. JCl Insight, 2.

27 Bedi, K.C., Jr., Snyder, N.W., Brandimarto, J., Aziz, M., Mesaros, C., Worth, A.J., Wang, L.L., Javaheri, A., Blair, I.A., Margulies, K.B. et al. (2016) Evidence for Intramyocardial Disruption of Lipid Metabolism and Increased Myocardial Ketone Utilization in Advanced Human Heart Failure. Circulation, 133, 706-716.

28 Martin, A.S., Abraham, D.M., Hershberger, K.A., Bhatt, D.P., Mao, L., Cui, H., Liu, J., Liu, X., Muehlbauer, M.J., Grimsrud, P.A. et al. (2017) Nicotinamide mononucleotide requires SIRT3 to improve cardiac function and bioenergetics in a Friedreich's ataxia cardiomyopathy model. JCI Insight, 2.

29 Nakai, A., Yamaguchi, O., Takeda, T., Higuchi, Y., Hikoso, S., Taniike, M., Omiya, S., Mizote, I., Matsumura, Y., Asahi, M. et al. (2007) The role of autophagy in cardiomyocytes in the basal state and in response to hemodynamic stress. Nat Med, 13, 619-624.

30 Bjorkoy, G., Lamark, T., Pankiv, S., Overvatn, A., Brech, A. and Johansen, T. (2009) Monitoring autophagic degradation of p62/SQSTM1. Methods Enzymol, 452, 181-197.

31 Linke, W.A. (2008) Sense and stretchability: the role of titin and titin-associated proteins in myocardial stress-sensing and mechanical dysfunction. Cardiovasc Res, 77, 637-648. 
32 Saxton, R.A. and Sabatini, D.M. (2017) mTOR Signaling in Growth, Metabolism, and Disease. Cell, 169, 361-371.

33 Guan, J., Mishra, S., Qiu, Y., Shi, J., Trudeau, K., Las, G., Liesa, M., Shirihai, O.S., Connors, L.H., Seldin, D.C. et al. (2014) Lysosomal dysfunction and impaired autophagy underlie the pathogenesis of amyloidogenic light chain-mediated cardiotoxicity. EMBO Mol Med, 6, 1493-1507.

34 Bishop, S.P. and Altschuld, R.A. (1970) Increased glycolytic metabolism in cardiac hypertrophy and congestive failure. Am J Physiol, 218, 153-159.

35 Allard, M.F., Schonekess, B.O., Henning, S.L., English, D.R. and Lopaschuk, G.D. (1994) Contribution of oxidative metabolism and glycolysis to ATP production in hypertrophied hearts. Am J Physiol, 267, H742-750.

36 Karamanlidis, G., Lee, C.F., Garcia-Menendez, L., Kolwicz, S.C., Jr., Suthammarak, W., Gong, G., Sedensky, M.M., Morgan, P.G., Wang, W. and Tian, R. (2013) Mitochondrial complex I deficiency increases protein acetylation and accelerates heart failure. Cell Metab, 18, 239-250.

37 Sutherland, F.J. and Hearse, D.J. (2000) The isolated blood and perfusion fluid perfused heart. Pharmacol Res, 41, 613-627.

\section{Legends to Figures}

Figure 1. Decreased autophagic degradation in hearts from 12 or 48 week old TTNtv rats. (A) Immunoblot and densitometric analysis of LC3B-II and p62 in left ventricle from 12 week old WT (n=3), TTNtvA ( $\mathrm{n}=4)$, and TTNtvZ (n=3) animals. (B-C) mRNA expression of Map1lc3b (B) and Sqstm1 (C) in left ventricle from 12 week old WT $(n=8)$, TTNtvA $(n=3)$, and TTNtvZ $(n=5)$ animal. (D) Immunoblot and densitometric analysis of LC3B-II and p62 in left ventricle from 48 week old WT (n=4), TTNtvA (n=4), and TTNtvZ $(\mathrm{n}=4)$ animals. Data is shown as mean \pm SEM. $* \mathrm{P}<0.05$. $* * \mathrm{P}<0.01$. Statistical testing was performed using one-way ANOVA.

Figure 2. Accumulation of p62 protein and autophagosomes in cardiomyocytes with TTNtv. (A) Representative image of immunochemistry staining of p62 in left ventricle from 12 week old WT, TTNtvA, and TTNtvZ animals. (B-C) Electron microscopy images of cardiomyocytes with autophagic vesicles labelled by white arrowheads (TTNtVA and TTNTvZ) (B) and bar graph (C) showing number of autophagic vesicles in WT, TTNtvA, and TTNtvZ based on EM micrograph images. Scoring was done by counting $>14$ different fields per condition. Data are shown as mean $\pm \mathrm{SEM},{ }^{*} \mathrm{P}<0.05, * * \mathrm{P}<0.01$, $* * * \mathrm{P}<0.001$ Statistical testing was performed using one-way ANOVA. 
Figure 3. Protein levels of cathespin B and D in TTNtv hearts. (A) Immunoblot and (B-E) densitometric analysis of cathepsin B and cathepsin D in left ventricle from 12 week old WT (n=3), TTNtvA (n=4), and $\operatorname{TTNtvZ}(\mathrm{n}=3)$ animals. Data are shown as mean $\pm \mathrm{SEM}, * \mathrm{P}<0.05$, $* * \mathrm{P}<0.01, * * * \mathrm{P}<0.001$ Statistical testing was performed using one-way ANOVA.

Figure 4. MTORC1 inhibitor rapamycin restored autophagic flux in TTNtv hearts. Rapamycin $(2 \mathrm{mg} / \mathrm{kg}$, dissolved in PBS containing $0.2 \%$ carboxymethyl cellulose and $0.25 \%$ tween 20 ) was injected intraperitoneally to TTNtv and WT rats for 7 consecutive days. Immunoblot and densitometric analysis of p-MTOR and MTOR (A-B), p62 and LC3B-II (C-D) in left ventricle from WT (n=4), TTNtvA (n=4), and TTNtvZ $(\mathrm{n}=4)$ treated with rapamycin or vehicle control. Data are shown as mean $\pm \mathrm{SEM}, * \mathrm{P}<0.05$, $* * \mathrm{P}<$ 0.01, Statistical testing was performed using one-way ANOVA.

Figure 5. Knockdown of titin decreased autophagic flux in H9C2 cells. mRNA was harvested $72 \mathrm{~h}$ after H9C2 cells were transfected with negative or titin siRNA. (A) mRNA level of titin was determined by quantitative RT-PCR. Six repearts are represented as mean \pm SEM. $* \mathrm{P}<0.05$. Statistical testing was performed using Student t test. (B-C) Immunoblot and densitometric analysis of LC3B-II in cells transfected with titin or negative siRNA with or without Bafilomycin A1. Bafilomycin A1-induced accumulation of LC3B-II was significantly decreased in titin knockdown cells. Triplicates are shown as mean \pm SEM. ${ }^{*} \mathrm{P}<0.05$. Statistical testing was performed using Student $t$ test. (D-E) Representative image and quantification of autophagosomes (yellow puncta) and autolysosomes (red puncta) in H9C2 cells transfected with negative or titin siRNA. Scoring was done by counting $>16$ different fields per condition. Bars represent the mean \pm SEM Statistical testing was performed using Student t test.

Figure 6. TTNtv impairs mitochondrial function in cardiomyocytes. (A-C) Seahorse analysis of oxygen consumption in isolated cardiomyocytes from 12 week old WT $(n=6)$, TTNtvA $(n=3)$, and TTNtvZ $(n=3)$ rat. Bar graphs showing relative basal OCR $(\mathbf{B})$, maximum OCR $(\mathbf{C})$. Experimental data were normalized against cell viability. Data are shown as mean $\pm \mathrm{SD} ; * * \mathrm{P}<0.01, * * * \mathrm{P}<0.001$. Statistical testing was performed using one-way ANOVA. 
Figure 7. Increased acetylation of mitochondrial proteins in TTNtv rat hearts. (A-B) Immunoblot and densitometric analysis showing acetylation of mitochondrial proteins in mitochondrial fraction isolated from left ventricle of 12 week old WT ( $n=4$ or 5), TTNtvA $(n=4)$, and TTNtvZ rat $(n=5)$. Data are shown as mean $\pm \mathrm{SEM} ; * \mathrm{P}<0.05$. Statistical testing was performed using student $\mathrm{t}$ test. $(\mathbf{C})$ Acetyl-CoA in the ventricle from WT and TTNtv rats. (D) NAD $/ \mathrm{NADH}$ ratio in the ventricle from $\mathrm{WT}(\mathrm{n}=6)$, TTNtvA (n=6), and TTNtvZ $(n=6)$ rats.

\author{
Abbreviations \\ TTNtv - Titin-truncating variants \\ DCM - dilated cardiomyopathy \\ OCR - oxygen consumption rate \\ ROS - reactive oxygen species
}



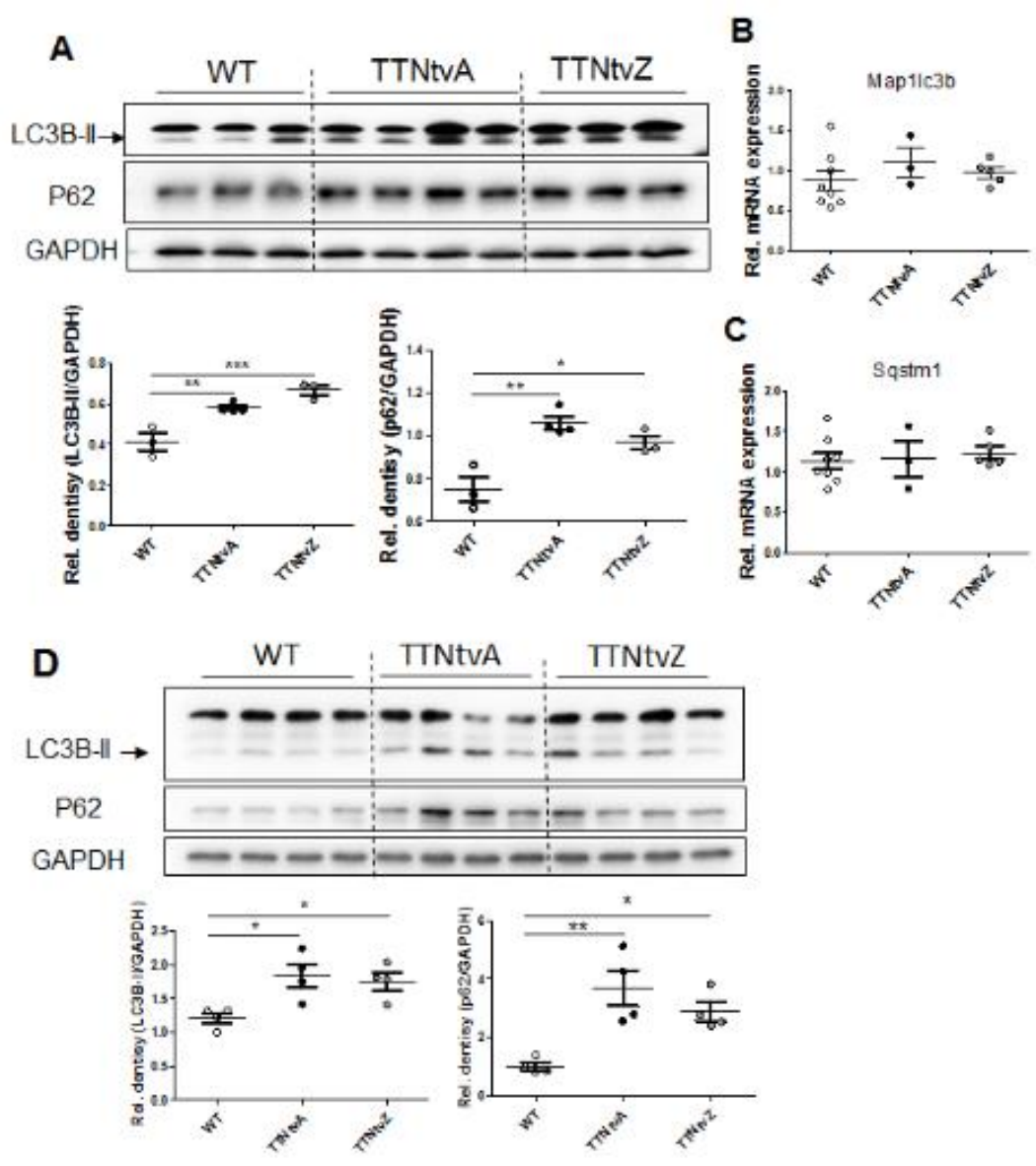

Figure 1. 


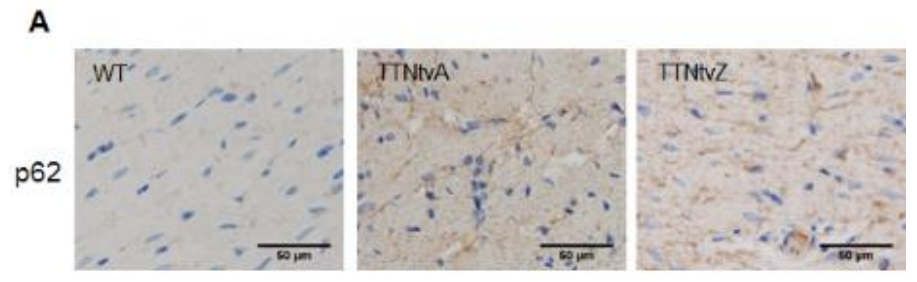

B
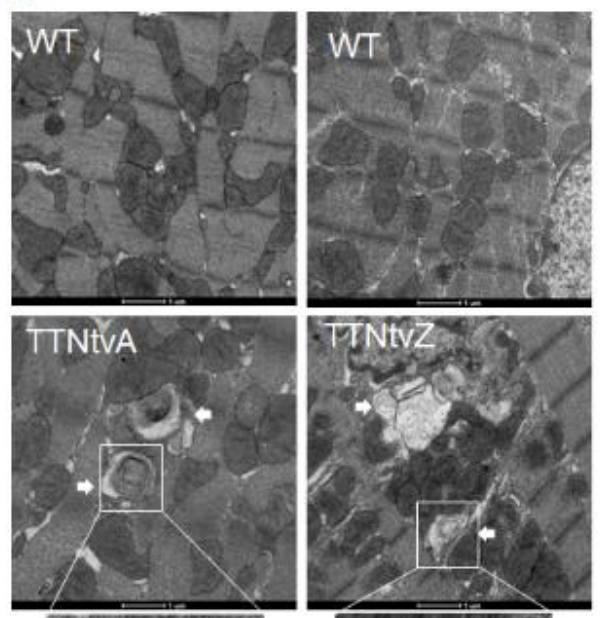

C
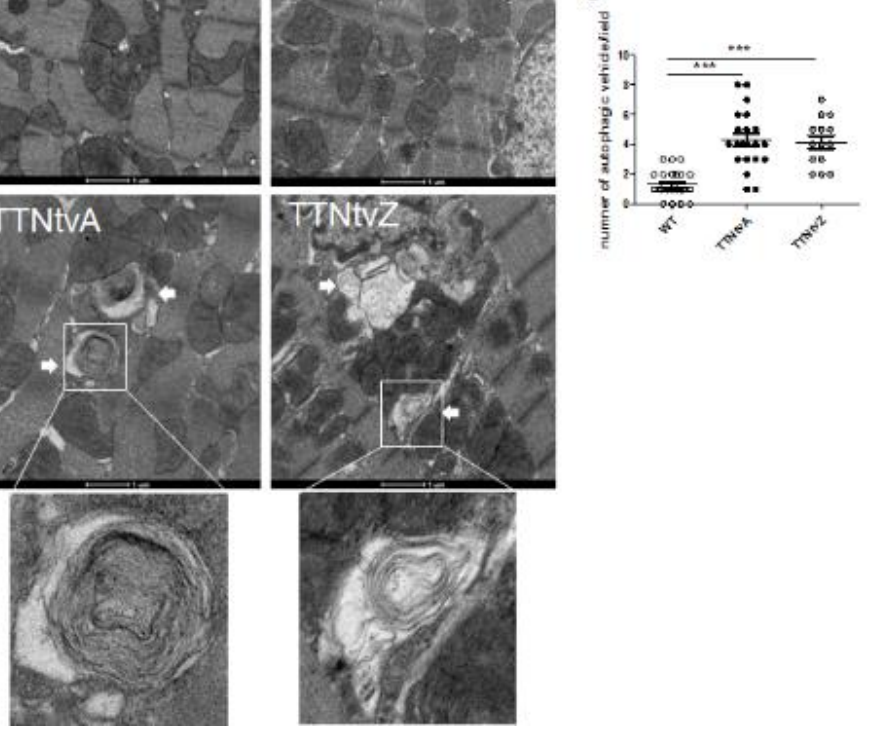

Figure 2. 


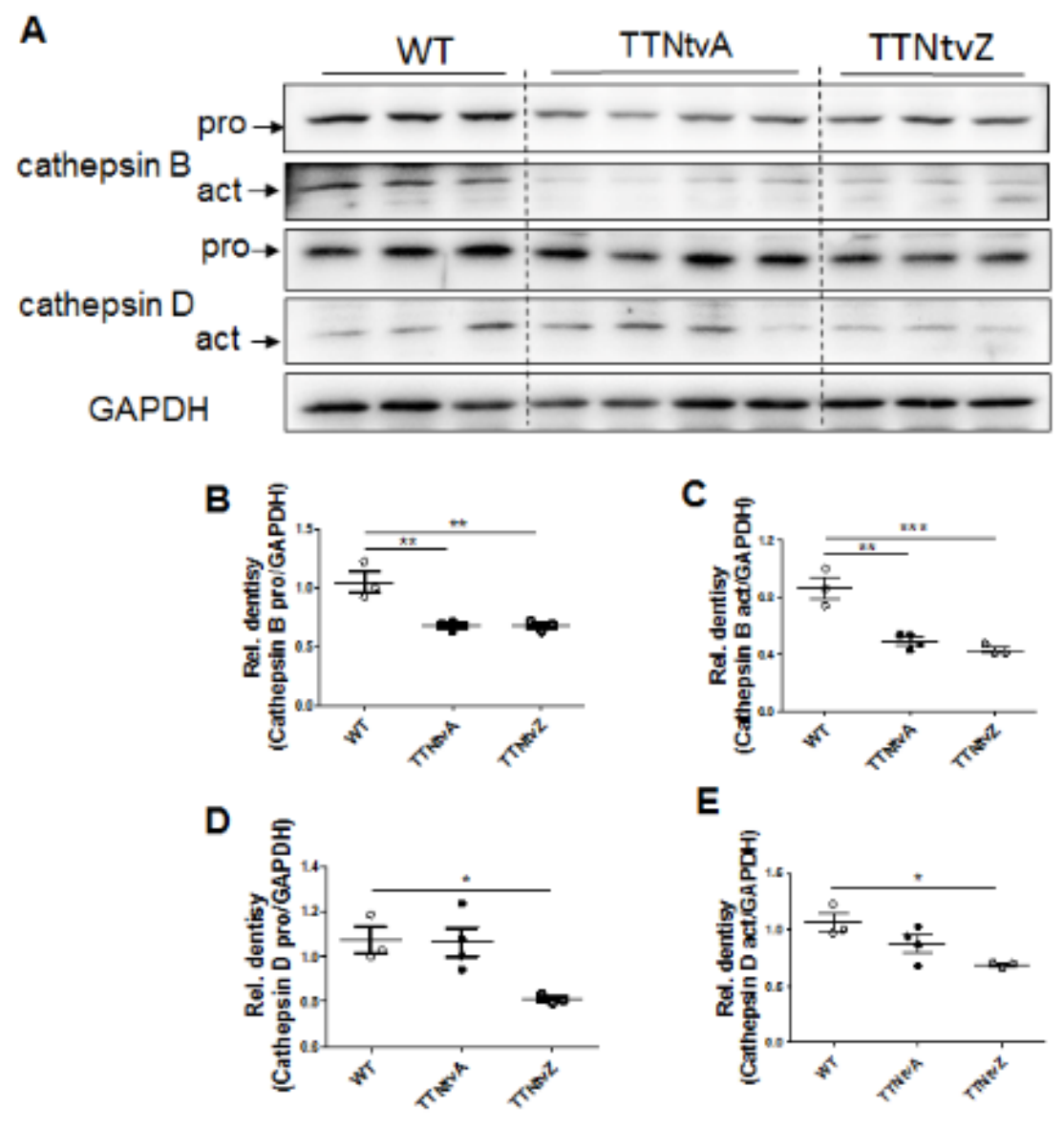

Figure 3. 


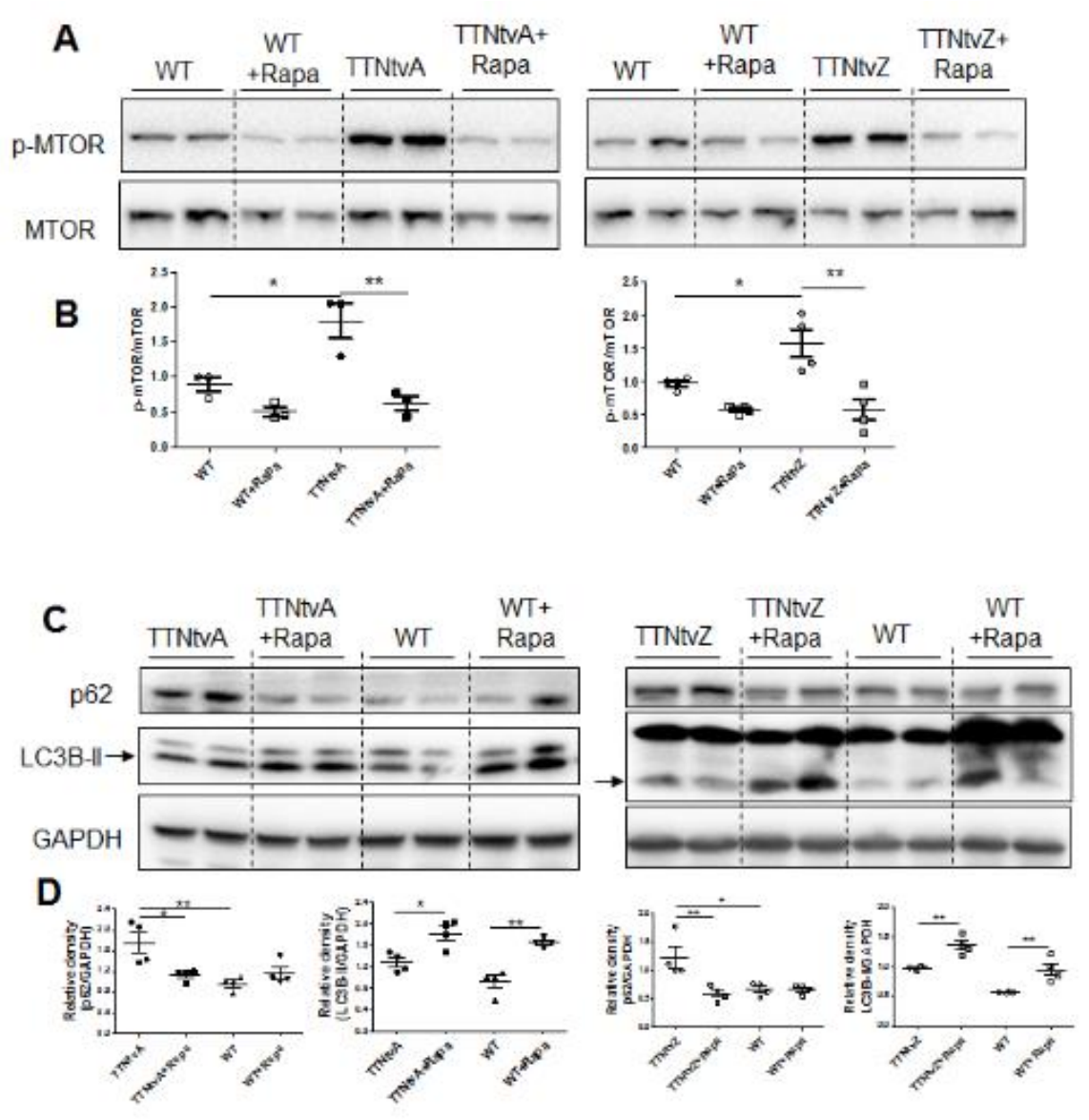

Figure 4. 

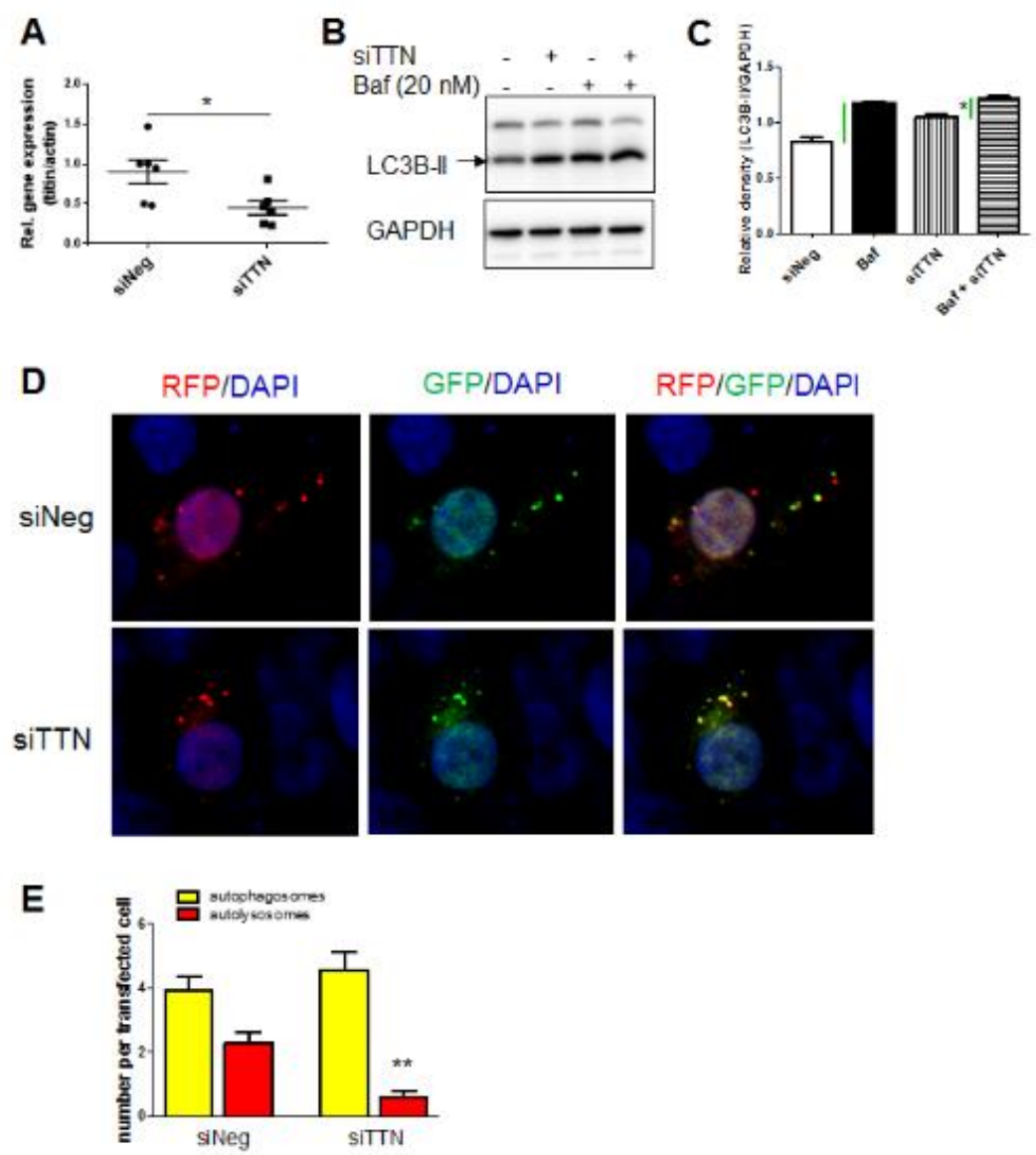

Figure 5. 

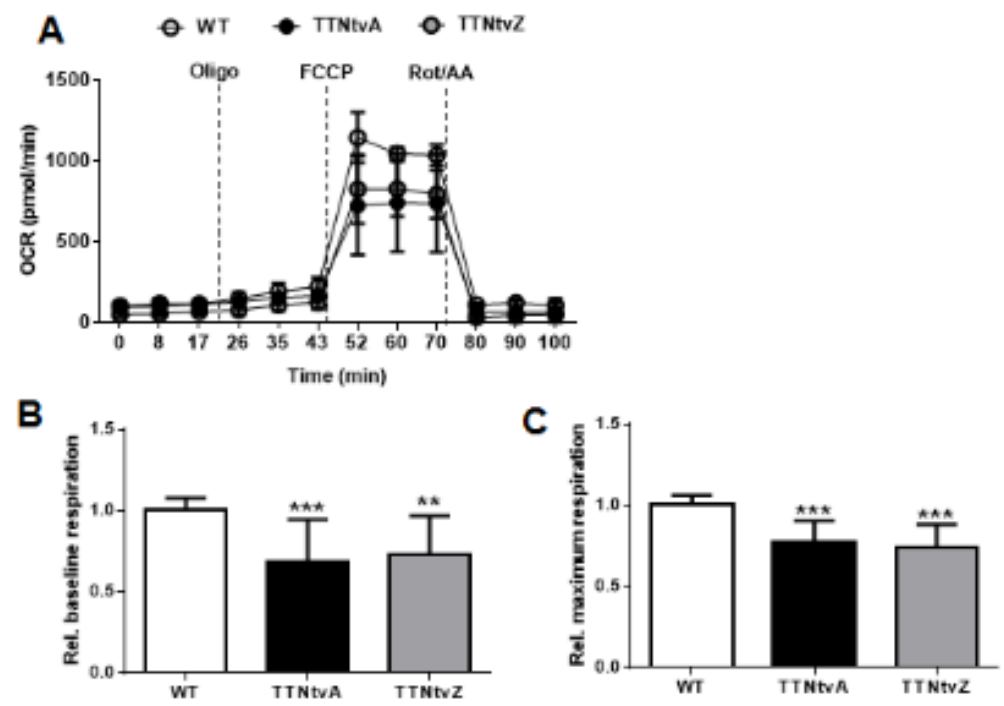

Figure 6. 


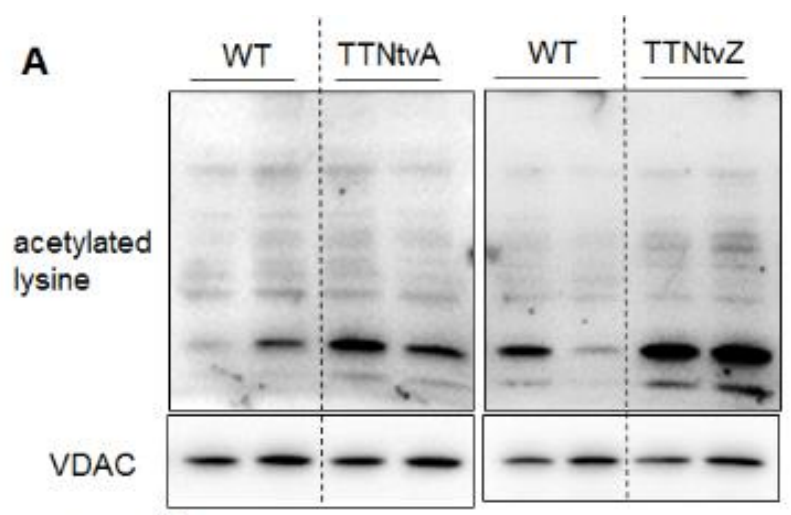

B
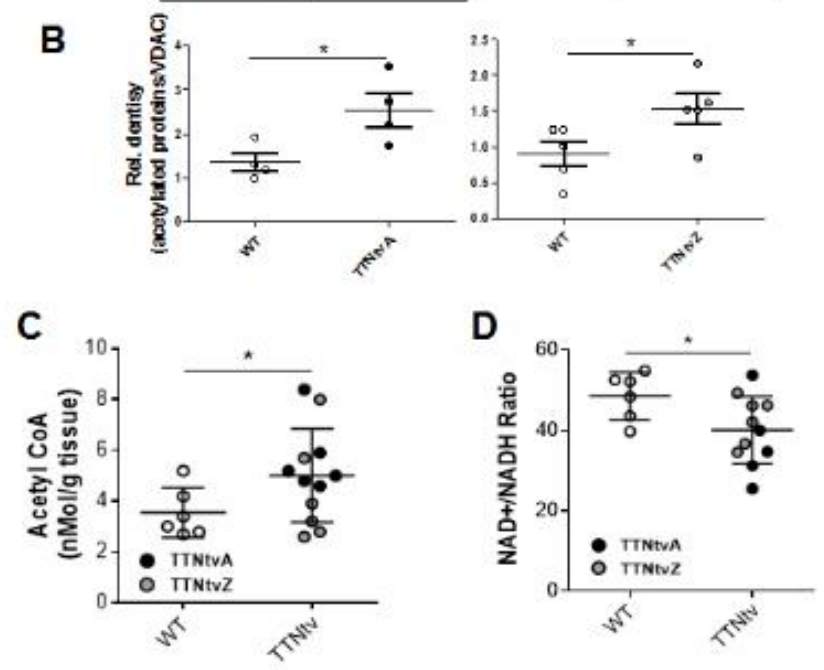

Figure 7. 


\section{Supplementary information}

Titin truncations lead to impaired cardiomyocyte autophagy and mitochondrial function in vivo

Jin Zhou ${ }^{1 \#}$, Benjamin $\mathrm{Ng}^{1,2 \#}$, Nicole S J Ko ${ }^{1}$, Lorna R Fiedler ${ }^{1}$, Ester Khin ${ }^{1}$, Andrea Lim ${ }^{1}$, Norliza E Sahib $^{1}$, Yajun $\mathrm{Wu}^{3}$, Sonia P Chothani ${ }^{1}$, Sebastian Schafer ${ }^{1,2}$, Boon-Huat Bay ${ }^{3}$, Rohit A. Sinha ${ }^{1,4}$, Stuart A. Cook ${ }^{1,2,5}$, Paul M. Yen ${ }^{1,6^{*}}$

${ }^{1}$ Cardiovascular \& Metabolic Disorders Program, Duke-National University of Singapore, Singapore 169857, Singapore.

${ }^{2}$ National Heart Centre Singapore, Singapore 169609, Singapore.

${ }^{3}$ Department of Anatomy, Yong Loo Lin School of Medicine, National University of Singapore, Singapore 117594, Singapore

${ }^{4}$ Department of Endocrinology, Sanjay Gandhi Postgraduate Institute of Medical Sciences, Lucknow226014, India.

${ }^{5}$ National Heart and Lung Institute, Imperial College London, London SW7 2AZ, UK.

${ }^{6}$ Duke Molecular Physiology Institute, Departments of Medicine and Pharmacology and Cancer Biology, Duke University Medical Center, Durham, NC 27710, USA.

\section{*Correspondence should be addressed to:}

Paul M. Yen (paul.yen@ duke-nus.edu.sg), Cardiovascular and Metabolic Disorders

Program, Duke-NUS Medical School, 8 College Road, Singapore 169857

\section{SINGAPORE.}

Tel: +65 65167332

Fax: +65 65348632 
\# The authors wish it to be known that, in their opinion, the first 2 authors should be regarded as joint First Authors.

\section{Methods}

\section{Animal models}

Rat TTNtv models were generated as previously described (1), and maintained on a F344 background. For A-band (C-terminus) truncating variant (TTNtvA), 12bp were deleted and 2bp inserted (TA) at 228608-228619 to introduce a stop codon in exon 303 (Genomic NCBI Ref Seq: NC_005102.3) corresponding to exon 327 in the human sequence. For Z-disc (N-terminus) truncating mutation (TTNtvZ) was generated by deletion of exons 2 to 6 (5286bp deletion, coordinates 2323-7608) to introduce a frameshift.

Animal studies were conducted in accordance with the principles and procedures outlined in the National Institutes of Health Guide for the Care and Use of Laboratory Animals and were approved by the Institutional Animal Care and Use Committee (IACUC, 2013/SHS/844) at the Duke-National University of Singapore Medical School.

12-week old male rats were used for this study. For starvation studies, TTNtv and WT rats were deprived of food for 14 hours, but had free access to water.

\section{RNA-seq data analysis}

For analysis of TFEB, CTSD, and LAMP1 mRNA expression in left ventricular tissue samples from human DCM patients, a previously published RNA-seq dataset was used (2). The dataset contains 105 control samples, 97 patients with end-stage DCM, including 7 patients with TTNtv. Differential expression analysis was carried out using DESeq2 (V1.18.1) (3). Pairwise comparison by wald test was carried out between all DCM patient samples $(n=97)$ and control samples, as well as DCM patients with TTNtv $(n=7)$ versus control samples. Boxplot and beeswarm R packages were used to visualize the genes of interest. 


\section{Cellular oxygen consumption rate (OCR) analysis in $\mathrm{H9C} 2$ cells}

Oxygen consumption was measured at $37^{\circ} \mathrm{C}$ using an XF24 extracellular analyzer (Seahorse Bioscience Inc., North Billerica, MA). H9C2 cells were seeded in 24-well plates and transfected with either negative or titin siRNA. Seventy-two hours after transfection, mitochondrial metabolic parameters were assessed using Mitostress test kit (Seahorse Biosciences, Cat. 103015-100). H9C2 cells were subsequently treated with Oligomycin $(1 \mu \mathrm{M})$, followed by FCCP $(2 \mu \mathrm{M})$ and lastly Rotenone/Antimycin A (1 $\mu \mathrm{M})$. Every point represents an average from four different wells.

\section{In vivo protein oxidation assay}

Frozen left ventricle tissue (20 mg) was minced and lysed in RIPA buffer containing protease and phosphatase inhibitors and $50 \mathrm{mM}$ DTT. The protein lysate was used for measurement of protein carbonyl formation as a readout for oxidative stress using an OxyBlot Protein Oxidation Detection Kit (Millipore, S7150) according to the manufacturer's instructions. 
Figure and Figure legend

\section{Supplementary Figure 1.}
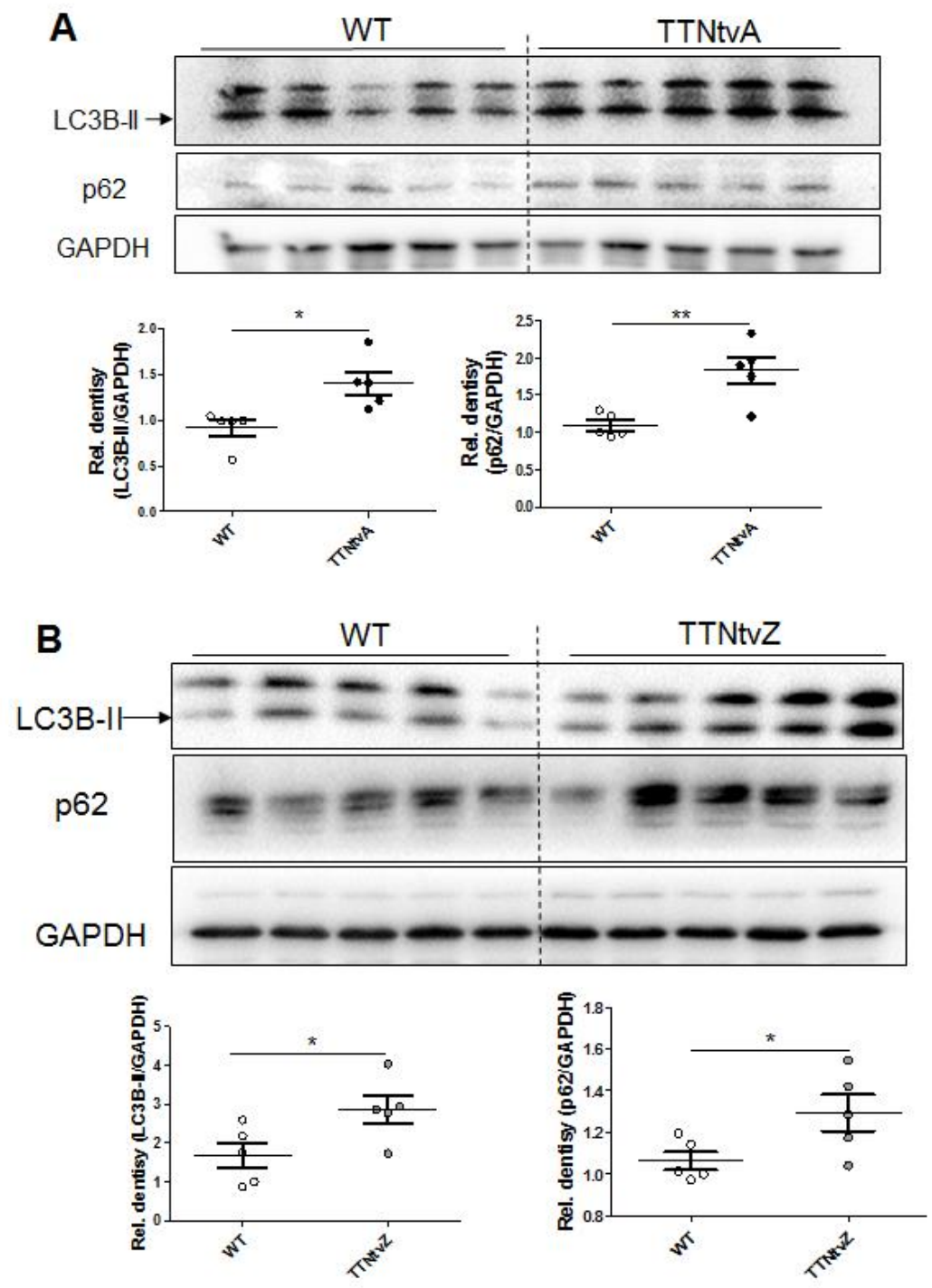

Supplementary Figure 1. Increased levels of LC3B-II and p62 in hearts from 12 weeks old

fasted TTNtv rats. TTNtv and WT rats were deprived of food for 14 hours, but had free access to water. (A) Immunoblot and densitometric analysis of LC3B-II and p62 in left ventricle from WT (n=5), and TTNtvA (n=5) animals. (B) Immunoblot and densitometric analysis of LC3B-II and p62 in left ventricle from WT $(n=5)$, and TTNtvZ $(n=5)$ animals. Data is shown as mean \pm SEM. ${ }^{*} \mathrm{P}<0.05$. Statistical testing was performed using Student $\mathrm{t}$ test. 


\section{Supplementary Figure 2.}
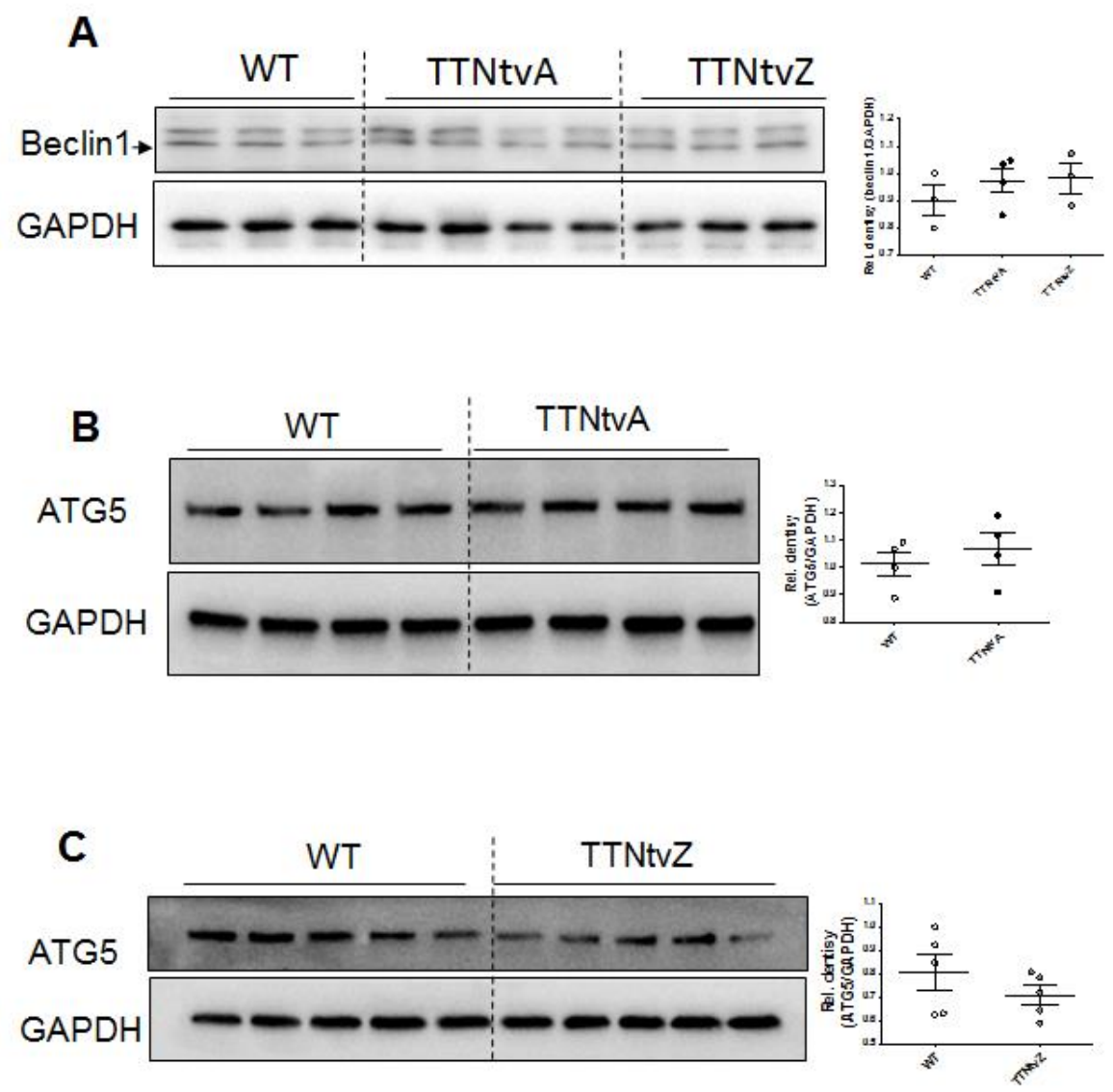

Supplementary Figure 2. Protein expression of Beclin1, and ATG5 in hearts from 12 weeks old TTNtv animals. (A) Immunoblot and densitometric analysis of Beclin 1 in left ventricle from WT ( $n=3)$, TTNtvA $(n=4)$, and TTNtvZ $(n=3)$ animals. Statistical testing was performed using one-way ANOVA. (B) Immunoblot and densitometric analysis of ATG5 in left ventricle from WT $(\mathrm{n}=4)$ and TTNtvA $(\mathrm{n}=4)$ animals. Statistical testing was performed using Student t test. $(\mathbf{C})$ Immunoblot and densitometric analysis of ATG5 in left ventricle from WT (n=5) and TTNtvZ $(\mathrm{n}=5)$ animals. Statistical testing was performed using Student $\mathrm{t}$ test. Data is shown as mean \pm SEM. 


\section{Supplementary Figure 3.}

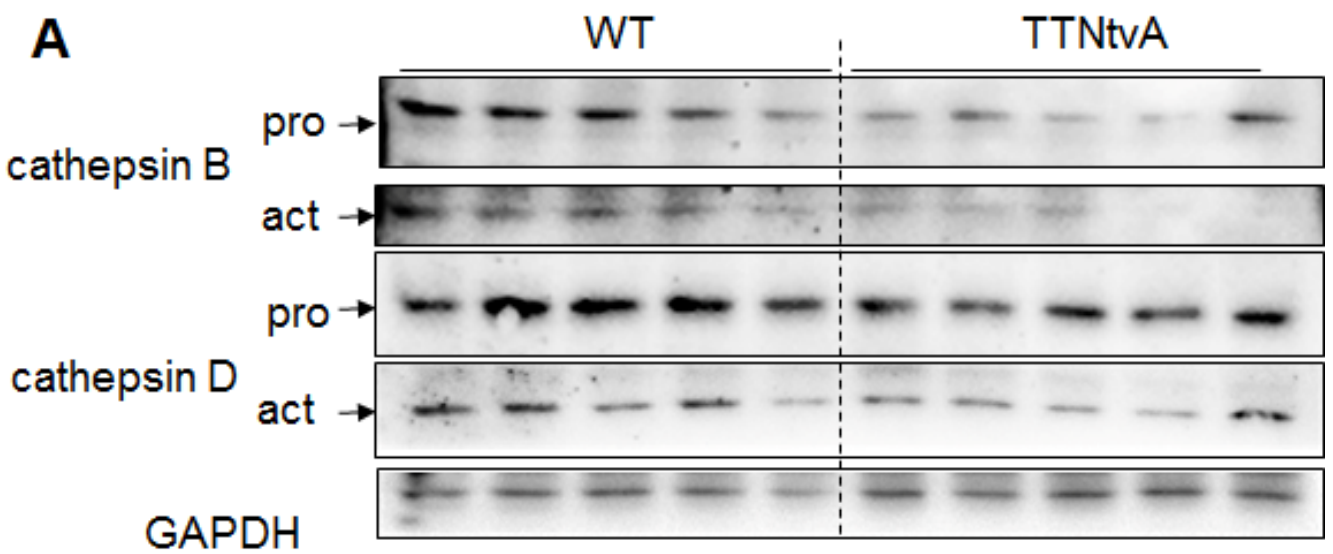

B

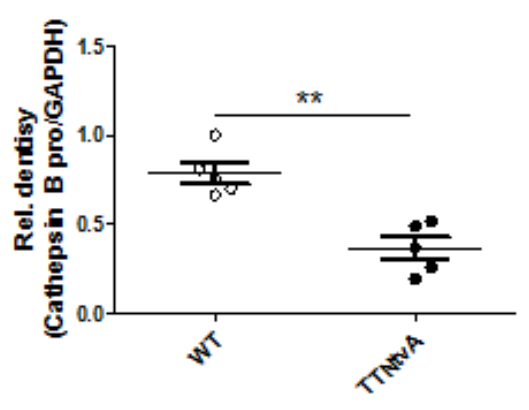

D

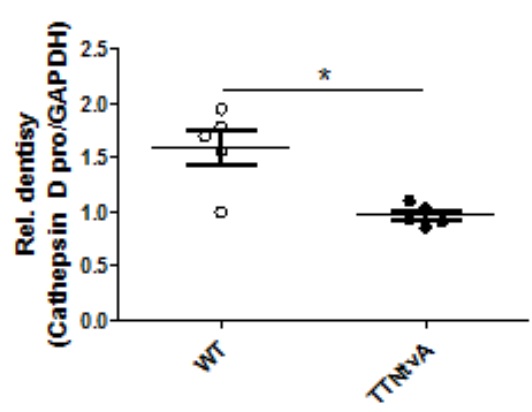

C
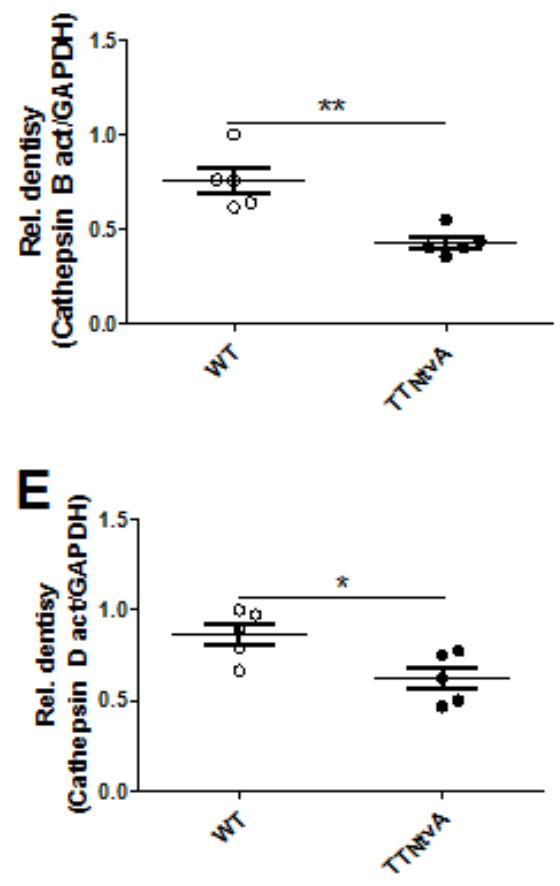

Supplementary Figure 3. Decreased levels of cathepsin B and D in hearts from 12 weeks old fasted TTNtvA rats. (A) Immunoblot and (B-E) densitometric analysis of cathespin B and cathepsin D in WT $(n=5)$, or TTNtvA $(n=5)$ hearts after overnight fasting. Data is shown as mean \pm SEM. $* \mathrm{P}<0.05 .{ }^{*} \mathrm{P}<0.01$. Statistical testing was performed using Student $\mathrm{t}$ test. 


\section{Supplementary Figure 4.}
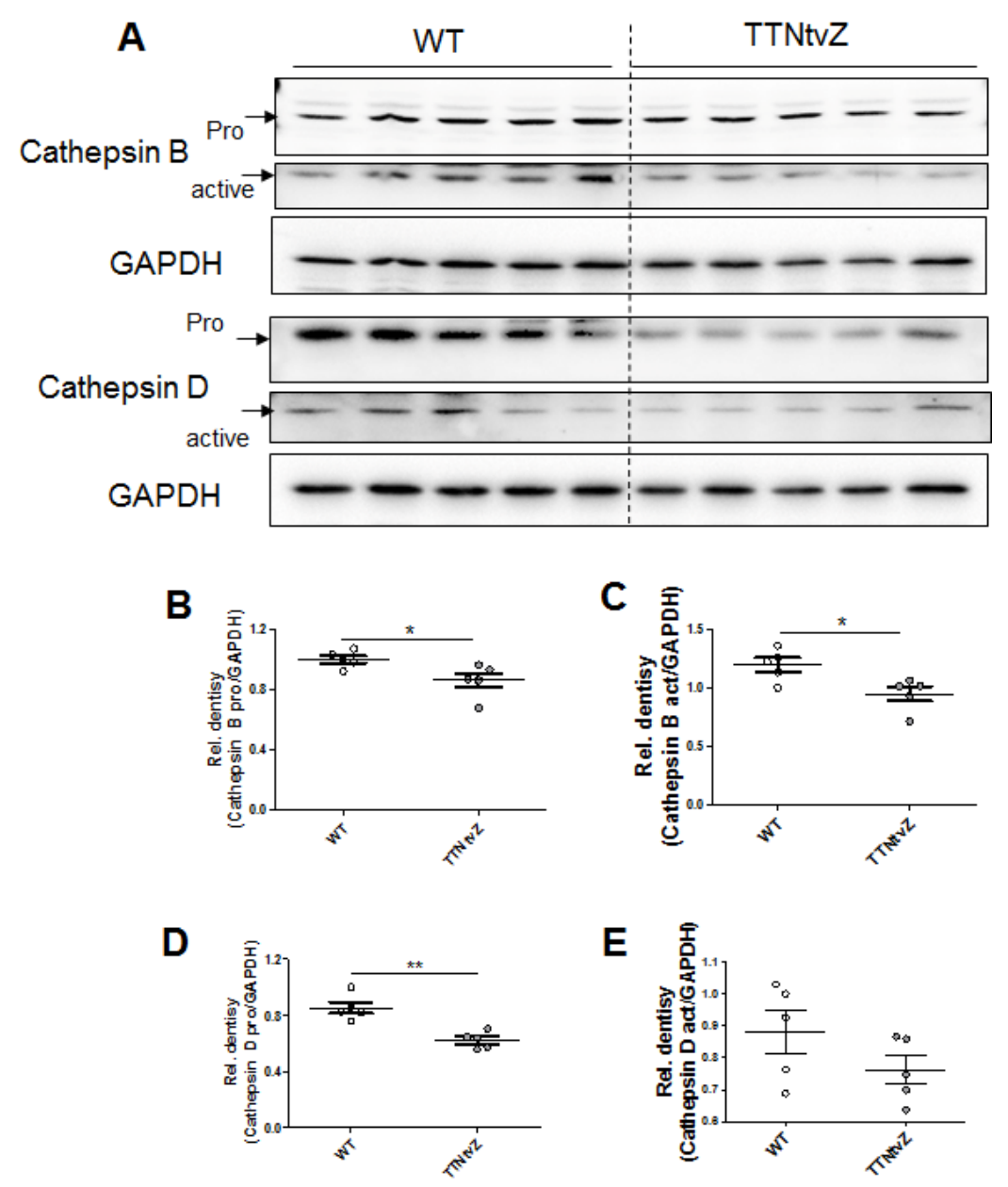

Supplementary Figure 4. Decreased levels of cathepsin B and D in hearts from 12 weeks old fasted TTNtvZ rats. (A) Immunoblot and (B-E) densitometric analysis of cathespin B and cathepsin D in WT $(n=5)$, or TTNtvZ $(n=5)$ hearts after overnight fasting. Data is shown as mean \pm SEM. ${ }^{*} \mathrm{P}<0.05$. ${ }^{* *} \mathrm{P}<0.01$. Statistical testing was performed using Student $\mathrm{t}$ test. 


\section{Supplementary Figure 5.}
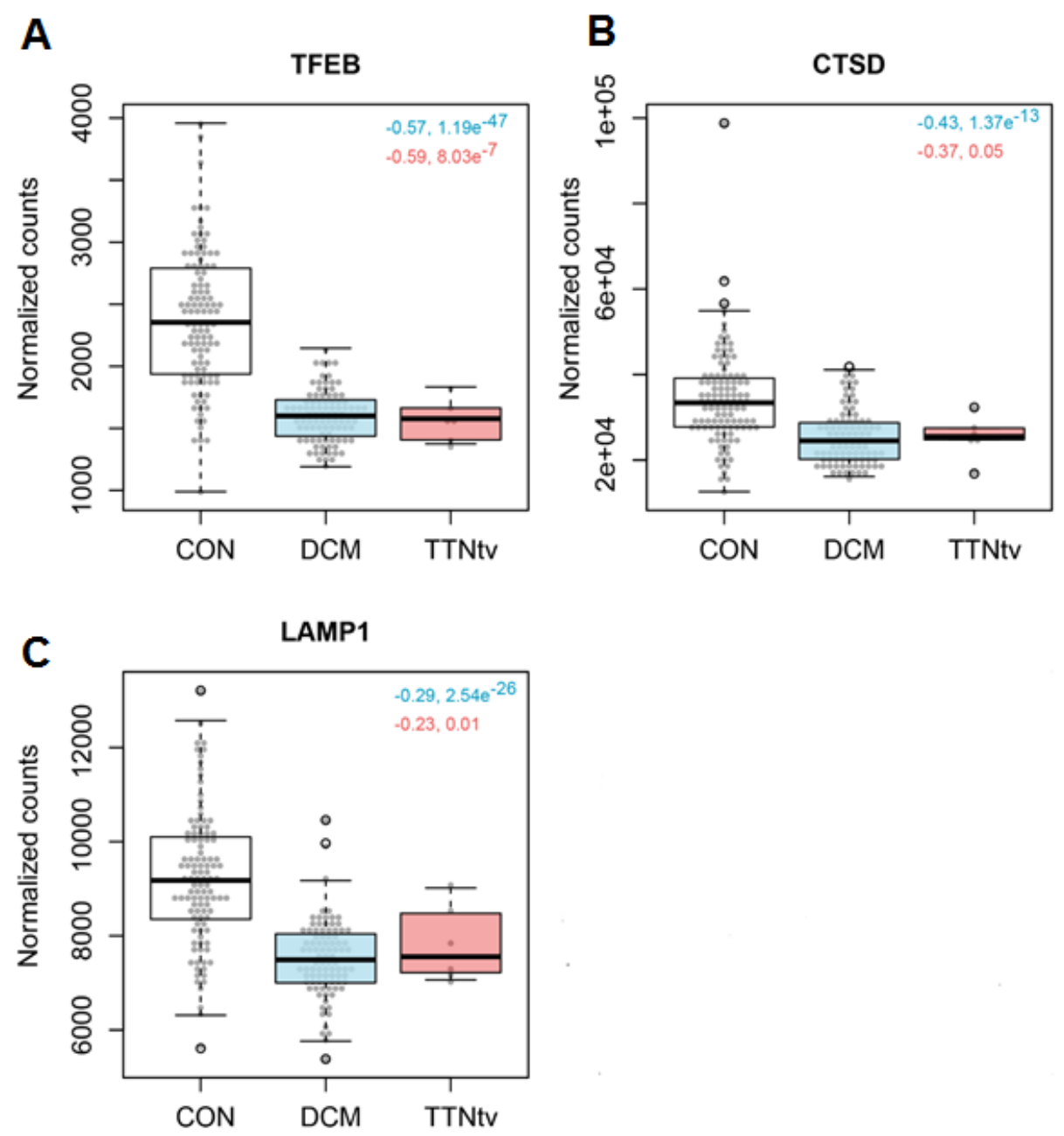

\section{Supplementary Figure 5. Decreased mRNA expression of lysosomal genes in DCM patients.}

Differential expression of published RNA-seq data containing DCM patients ( $\mathrm{n}=97$ ), DCM patients with TTNtv $(n=7)$ and non-diseased controls $(n=105)$ showed decreased mRNA level of (A) TFEB, (B) CTSD, and (C) LAMP1 in DCM patients and DCM patients with TTNtv. Boxplot and beeswarm $\mathrm{R}$ packages were used to visualize the genes of interest, with $\log _{2}$ fold change, followed by Benjamini-Hochberg adjusted p-value indicated in the plot. 


\section{Supplementary Figure 6.}

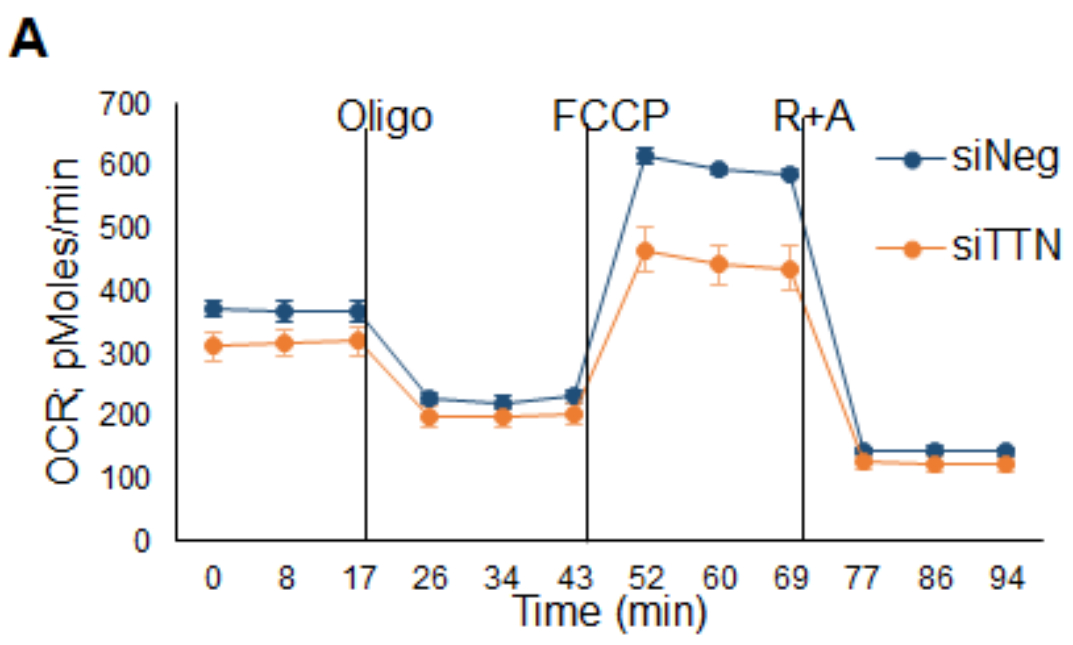

B
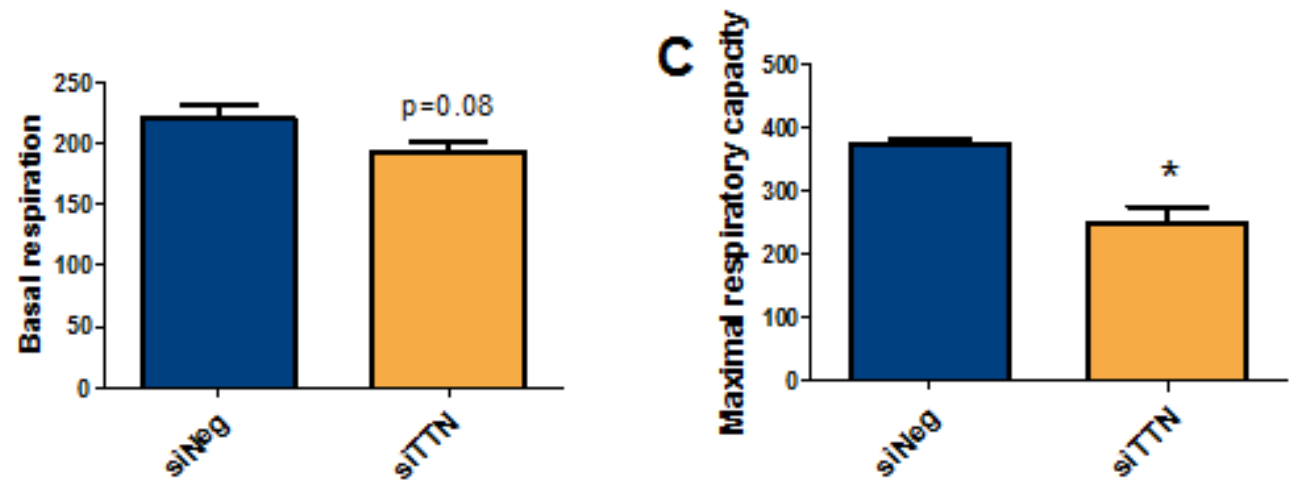

Supplementary Figure 6. Knockdown of titin decreased oxygen consumption (OCR) in H9C2

cells. $72 \mathrm{~h}$ after cells were transfected with negative or titin siRNA, OCR was measured using Mitostress test kit. Basal respiration (B), maximal respiratory capacity $(\mathbf{C})$, were calculated. Bars represent the mean value of 4 different wells with SEM. Statistical testing was performed using Student $t$ test. 


\section{Supplementary Figure 7.}
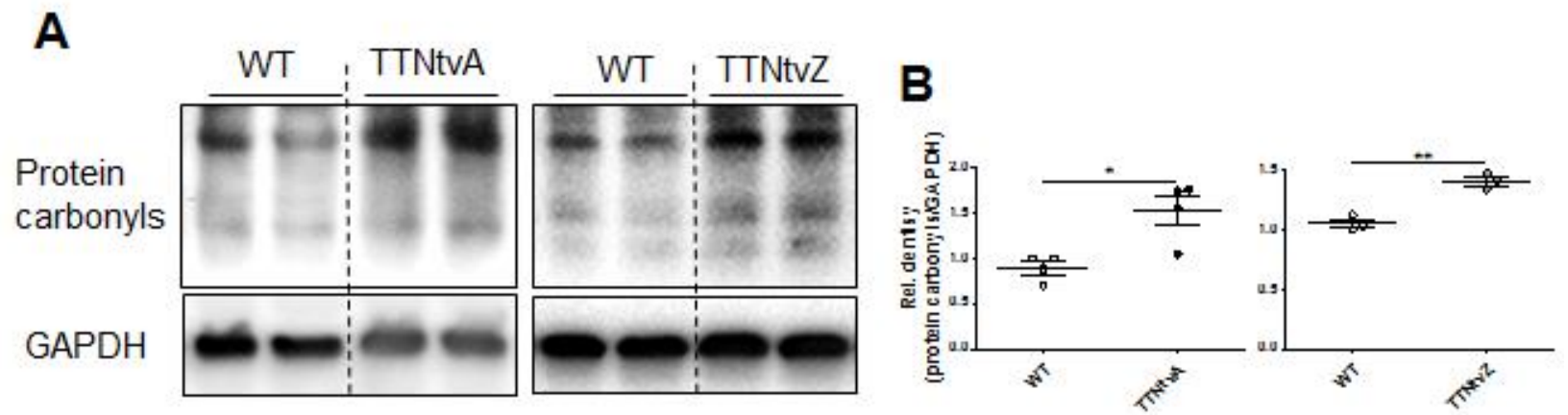

Supplementary Figure 7. TTNtv increased protein carbonyls in the heart. (A) Oxyblot and (B) densitometric analysis of protein carbonyls in the left ventricle of 12 week old animals. WT $(\mathrm{n}=4$ or 3$)$, TTNtvA $(\mathrm{n}=4)$, TTNtvZ $(\mathrm{n}=3)$. Data are shown as mean \pm SEM; $* \mathrm{P}<0.05, * * \mathrm{P}<0.01$. Statistical testing was performed using Student $\mathrm{t}$ test. 


\section{Supplementary Figure 8.}

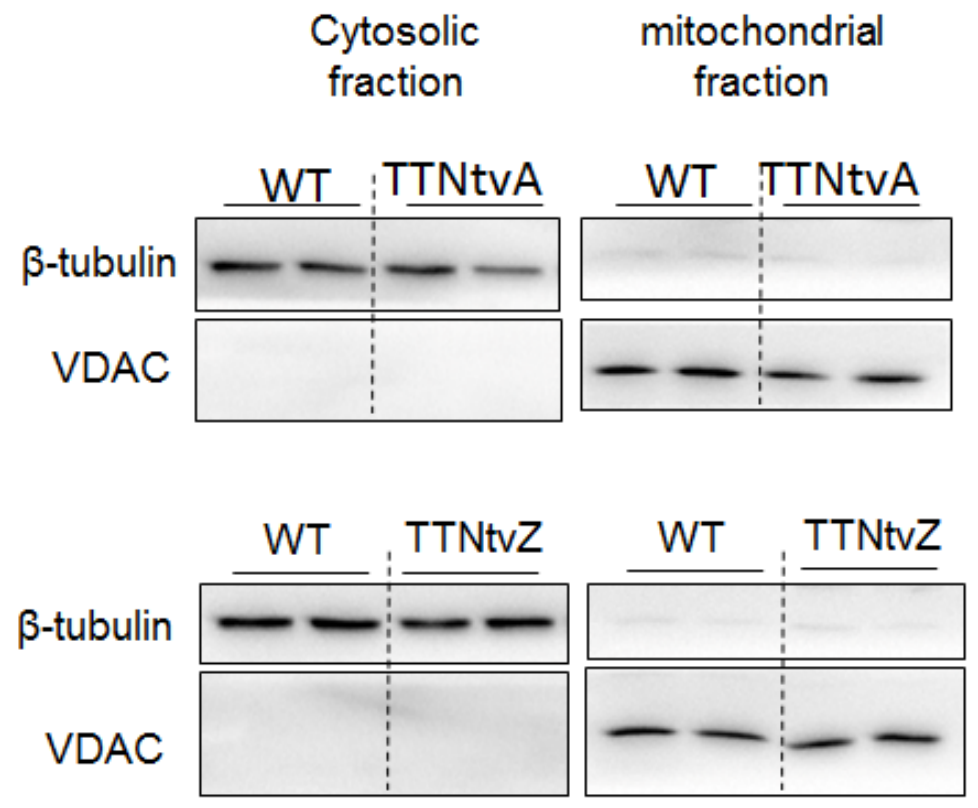

Supplementary Figure 8. Immunoblot showing purity/enrichment of the mitochondrial

fraction. Purity of mitochondrial was verified by the absence of $\beta$-tubulin (cytosolic), and purity of cytosolic fraction was verified by the absence of mitochondrial protein VDAC. 


\section{Supplementary Figure 9.}

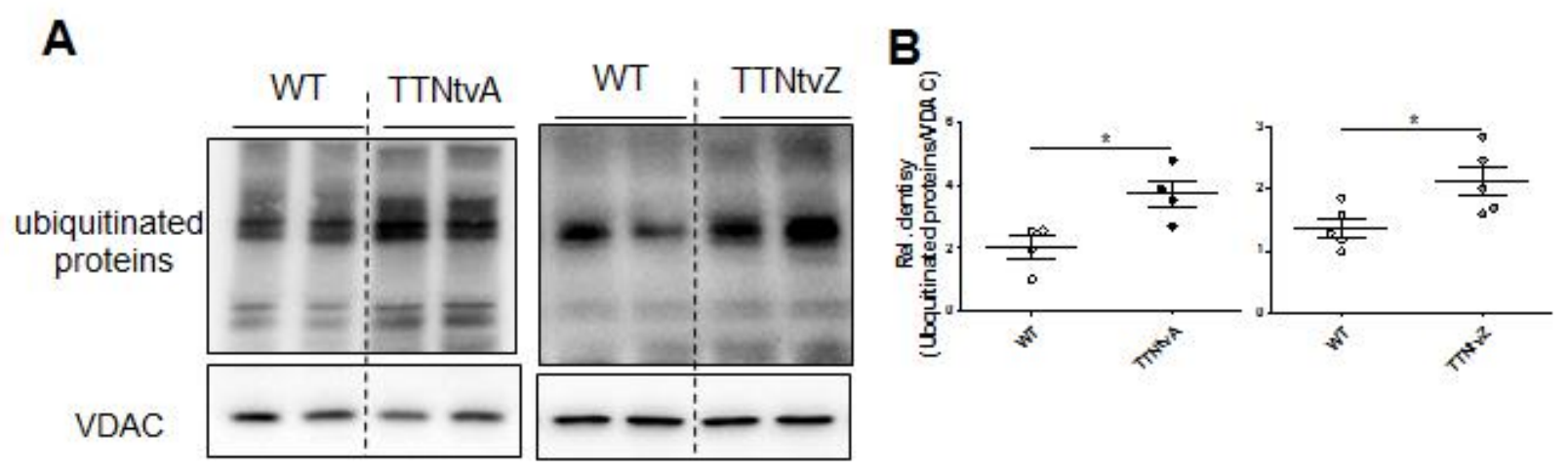

Supplementary Figure 9. TTNtv increased mitochondrial ubiquitination in the heart. (A) Immunoblot and (B) densitometric analysis showing ubiquitination of mitochondrial proteins in isolated mitochondrial fraction from left ventricle of WT ( $\mathrm{n}=4$ or 5), TTNtvA $(\mathrm{n}=4)$, and TTNtvZ $(\mathrm{n}=5)$ rat. Data are shown as mean $\pm \mathrm{SEM} ; * \mathrm{P}<0.05$. Statistical testing was performed using Student $\mathrm{t}$ test. 


\section{Supplementary Figure 10.}

A

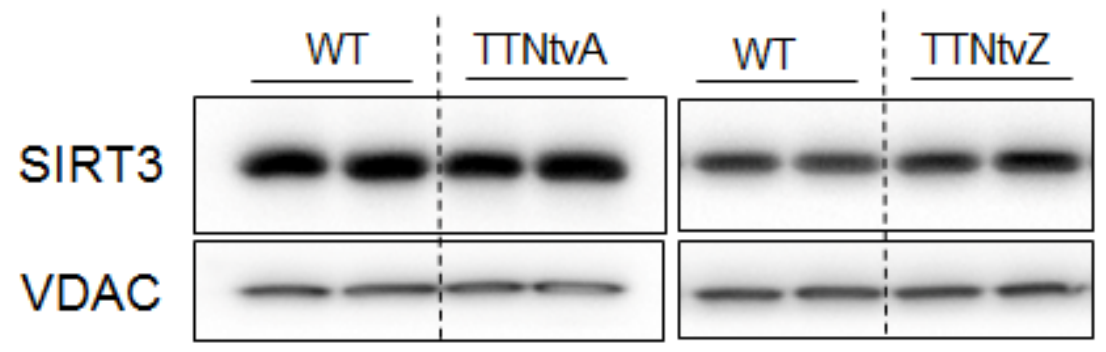

B

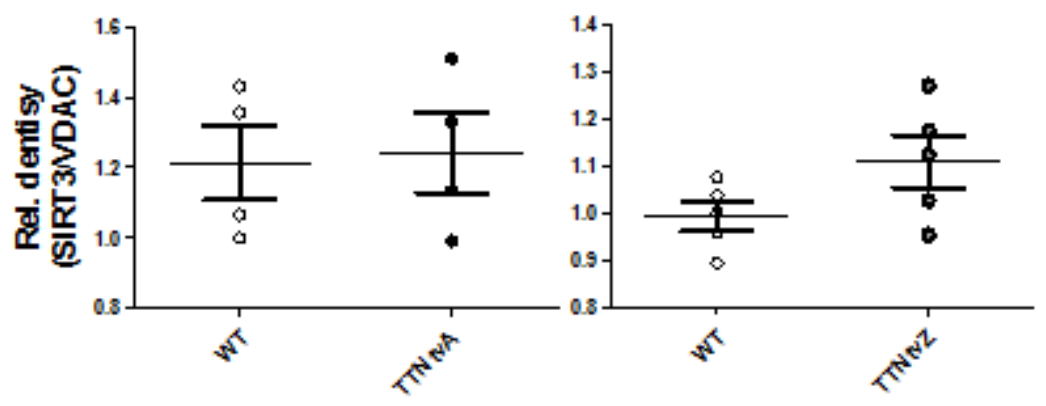

Supplementary Figure 10. No change of SIRT3 protein in mitochondrial fraction isolated

from TTNtv or WT rat hearts. (A-B) Immunoblot and densitometric analysis showing SIRT3 protein level in mitochondrial fraction isolated from left ventricle of WT $(n=4)$, TTNtvA $(n=4)$, and TTNtvZ rat $(\mathrm{n}=5)$. Data are shown as mean \pm SEM. Statistical testing was performed using student $t$ test.

\section{Reference}

1 Schafer, S., de Marvao, A., Adami, E., Fiedler, L.R., Ng, B., Khin, E., Rackham, O.J., van Heesch, S., Pua, C.J., Kui, M. et al. (2017) Titin-truncating variants affect heart function in disease cohorts and the general population. Nat Genet, 49, 46-53. 
2 Heinig, M., Adriaens, M.E., Schafer, S., van Deutekom, H.W.M., Lodder, E.M., Ware, J.S., Schneider, V., Felkin, L.E., Creemers, E.E., Meder, B. et al. (2017) Natural genetic variation of the cardiac transcriptome in non-diseased donors and patients with dilated cardiomyopathy. Genome Biol, 18, 170.

3 Love, M.I., Huber, W. and Anders, S. (2014) Moderated estimation of fold change and dispersion for RNA-seq data with DESeq2. Genome Biol, 15, 550. 